\title{
3D rock fabric analysis using micro-tomography: An introduction to the open-source TomoFab MATLAB code
}

\author{
Benoît Petri $^{\mathrm{a}, \mathrm{b}, 1, *}$, Bjarne S.G. Almqvist ${ }^{\mathrm{c}, 2}$, Mattia Pistone ${ }^{\mathrm{a}, \mathrm{d}, 3}$ \\ ${ }^{a}$ Institute of Earth Sciences, University of Lausanne, Geopolis, 1015, Lausanne, Switzerland \\ ${ }^{\mathrm{b}}$ Universite de Strasbourg, CNRS, IPGS UMR 7516, F-67000, Strasbourg, France \\ ${ }^{c}$ Department of Earth Sciences, Uppsala University, Villavagen 16, 752 36, Uppsala, Sweden \\ ${ }^{\mathrm{d}}$ Department of Geology, Franklin College of Arts and Sciences, University of Georgia, 210 Field Street, Athens, GA, 30602-2501, USA
}

\section{A R T I C L E I N F O}

\section{Keywords:}

Microfabric analysis

Tensor analysis

Micro-tomography

$\mathrm{X}$-ray computed tomography

MATLAB GUI

\begin{abstract}
A B S T R A C T
The study of rock fabric properties (orientation, planar, linear, anisotropy) is key to unravelling the geological processes that generated them. With advancements in data acquisition and treatment, X-ray micro-computed tomography $(\mu \mathrm{XCT})$ represents a powerful method to analyse the shape preferred orientation (SPO) of rockforming elements, including minerals, aggregates, and pores, in the three-dimensional space. After reconstruction and segmentation of $\mu \mathrm{XCT}$ images, we developed a novel protocol to construct and analyse the fabric tensor, a second-rank symmetric tensor constructed using the orientation and the length of the three characteristic axes of each grain (simplified to a best fit ellipsoid). The analysis of the fabric tensor permits calculation of mean principal directions and associated confidence ellipses, and quantifies the degree of anisotropy $\left(P^{\prime}\right)$ and the shape $(T)$ of the fabric ellipsoid by eigenvalue and eigenvector analysis.

We implement this method in the TomoFab open-source MATLAB package. The code integrates a graphical user interface (GUI) that allows the visualisation of the full set of ellipsoid orientation, shape, and size. Density plots and contouring can be utilised to identify fabrics graphically, and a full set of fabric parameters can be calculated based on the analysis of the fabric tensor and/or the analysis of each principal direction orientation tensor.

We demonstrate the versatility of TomoFab with synthetic datasets and a field- and laboratory-based investigation of a sample presenting a magmatic foliation and lineation, collected in the Mafic Complex within the lower crustal section of the Ivrea-Verbano Zone (North Italy). In the light of these developments, we stress that $\mu \mathrm{XCT}$ represents a pertinent tool for rock fabric analysis to characterise the SPO of rock components. This approach can be performed parallel or complementary to other rock fabric quantification methods (e.g., AMS, EBSD) and applied to various rock types. TomoFab is freely available for download at https://github. com/benpetri/tomofab.
\end{abstract}

\section{Introduction}

A rock fabric (or petrofabric) corresponds to a spatial and geometrical arrangement of elements inherent to a rock (minerals, grain aggregates, pores, inclusions). This is controlled by compositional layering, grain size variations of minerals, shape preferred orientation (SPO) and crystallographic preferred orientation (CPO) of grains. The study of rock fabrics provides multi-scale insights into various geological processes such as magma flow (Benn and Allard, 1989; Nicolas, 1992), rock strain patterns (Chopin et al., 2012), and paleo-sediment transport directions (Benn, 1994). The fabric development can be described semi-quantitatively in the field (foliation, lineation), but proper quantification requires more rigorous methods (Higgins, 2006). To date, quantitative fabric studies involve electron backscatter diffraction (EBSD; Prior et al., 1999) measurements for CPO determination (e.g., Bascou et al., 2001) and magnetic fabric measurements, such as

\footnotetext{
* Corresponding author. Universite de Strasbourg, CNRS, IPGS UMR7516, F-67000, Strasbourg, France.

E-mail address: bpetri@unistra.fr (B. Petri).

1 Development of ideas and software, data treatment, participation to discussions and manuscript writing.

${ }^{2}$ Development of ideas, participation to discussions and manuscript writing.

${ }^{3}$ Data acquisition and treatment, participation to discussions and manuscript writing.
} 

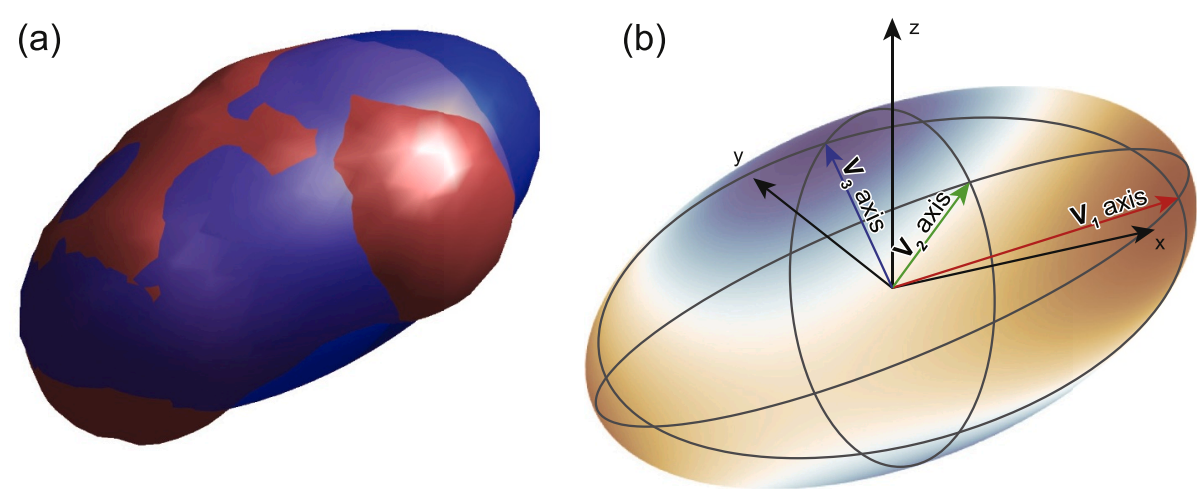

Fig. 1. (a) Three-dimensional view of a segmented grain (red) and its best-fit ellipsoid (blue), segmented and compiled with Blob3D (Ketcham, 2005b). (b) Three-dimensional view of the ellipsoid characterised by the length and orientation of three orthogonal principal axes $\mathbf{V}_{\mathbf{1}}, \mathbf{V}_{\mathbf{2}}$ and $\mathbf{V}_{\mathbf{3}}$. Colour map from Crameri (2018) representing the distance to the ellipsoid centre. (For interpretation of the references to colour in this figure legend, the reader is referred to the Web version of this article.)

anisotropy of both magnetic susceptibility (AMS) and anhysteretic remanent magnetization (AARM) (Borradaile and Jackson, 2010; Hirt and Almqvist, 2012); AMS and AARM depend on both CPO and SPO (for ferromagnetic minerals) of the magnetic minerals of rocks (Petri et al., 2018). Obtaining the three dimensional SPO is however time-consuming because it requires either serial sectioning or grinding of samples and image analysis (e.g., Bryon et al., 1995). Alternatively, the SPO can be estimated by analysing three or more section planes (slabs or thin sections) at high-angle to one another or by more advanced statistical methods if the observation planes are not mutually perpendicular (e.g., Heilbronner, 1992; Launeau and Robin, 2005; Moreno Chavez et al., 2018; Robin, 2002; Shan, 2008). The characterisation of a petrofabric by SPO requires determining the shape and orientation of each individual rock component. Consequently, a fabric can be either weakly developed (i.e., isotropic) with rounded and/or randomly oriented components or strongly developed (i.e., anisotropic) with highly elongated or flattened and strongly aligned components.

The development of micro-computed tomography techniques, using either X-ray or neutron tomography (e.g., Ketcham and Carlson, 2001; Vontobel et al., 2006), provides access to the three-dimensional exploration of a rock volume and represents a powerful tool when single grains and/or grain aggregates can be visualised and segmented. Micro-computed tomography has found several applications ranging from fluid flow characterisation in porous media (Islam et al., 2018; Starnoni et al., 2017) to imaging biological matter (Titschack et al., 2018). The size, shape and orientation of each individual object can be extracted (e.g., Som et al., 2013) using Blob3D for instance (Ketcham, 2005b), but this approach requires subsequent statistical treatment. There is a wealth of literature on the statistics of $3 \mathrm{D}$ orientation data (Fisher, 1953; Watson and Irving, 1957), which is summarised in several text books (Borradaile, 2003; Fisher et al., 1993; Mardia and Jupp, 2009). Specifically, spherical statistics are mainly obtained by means of vector (or axis) analysis or by analysing the orientation or the fabric second-rank tensors from which several parameters can be used to calculate the properties of the fabrics. While the construction of the orientation tensor is unequivocal (Scheidegger, 1965; Watson, 1966), various approaches to construct a "fabric tensor" have been reported, notably for bone structure characterisation (Odgaard, 1997) and applied to rock fabric quantification (Ketcham, 2005a; Launeau and Cruden, 1998; Launeau and Robin, 1996; Macente et al., 2017; Schopa et al., 2015). The different approaches to construct a fabric tensor are: (1) the star length distribution (SLD; Odgaard et al., 1997; Smit and Odgaard, 1998), which probes the grain shape by analysing the length of axes sourcing from a point located in the volume; (2) the star volume distribution (SVD), which reports the volume of a cone that originates from a point located in the volume; and (3) the mean intercept length (MIL; Harrigan and Mann, 1984; Whitehouse, 1974), which is determined using the number of intercepts between a linear grid and the considered volume surface. All these continuum methods apply a regularly or randomly distributed counting grid that is particularly appropriate for
Table 1

Summary of symbols used in the equations, text and figures.

\begin{tabular}{ll}
\hline Symbol & Definition \\
\hline $\mathrm{i}$ & Index of ellipsoid axis: 1, 2, 3 \\
$\mathrm{j}$ & Index of ellipsoid: from 1 to N \\
l, m, n & Directional cosines of an axis \\
$\mathrm{a}$ & Axis directional cosines in vector format: (1, m, n) \\
$\mathrm{N}$ & Number of ellipsoids considered \\
$\mathbf{V}$ & Axis coordinates in vector format \\
$\mathrm{R}$ & Length of the mean axis calculated from a population of axes \\
$\mathbf{O}$ & Orientation tensor calculated from directional cosines of a population of \\
& axes \\
$\mathbf{L}$ & Linear fabric tensor calculated from a set of axes triplet \\
$\mathbf{Q}$ & Quadratic fabric tensor calculated from a set of axes triplet \\
$\lambda$ & Eigenvalue of orientation or fabric tensors \\
$\mathrm{V}$ & Eigenvector of orientation or fabric tensors \\
$\mathrm{E}$ & Confidence angle around specified direction \\
$P, G, R$ & Point, Girdle, or Random orientation distribution parameters of Vollmer \\
& (1990) \\
$K$ & Or K-index; fabric shape parameter of Woodcock (1977) \\
$L S$ & Fabric shape parameter of Ulrich and Mainprice (2005) \\
$T$ & Fabric shape parameter of Jelínek (1981) \\
$P$, & Degree of anisotropy of a fabric of Jelínek (1981) \\
\hline
\end{tabular}

the characterisation of the fabric of a connected network of elements (e. g., connected pores). However, none of the current tools uses the full set of data that can be extracted from the best-fit ellipsoids that approximate grains and/or grain aggregates (Jerram and Higgins, 2007; Ketcham, 2005b, 2005a) with the aim of quantifying a fabric defined by a population of grains or pores.

We present a set of parameters used to quantify the fabric development as basis of the TomoFab MATLAB package, which we developed to visualise and quantify petro-fabrics using micro-tomographic data. We finally illustrate and discuss the methodology using synthetic datasets and a case study from a rock core of gabbro presenting a magmatic foliation and lineation, collected in the Ivrea-Verbano Zone (Southern Alps, North Italy).

\section{Methods}

To satisfy the mutual dependency of three orthogonal principal axis orientation distributions (and related confidence angles), an appropriate approach should use the shape and the orientation of each individual grains simultaneously (i.e., the direction and length of the three principal axes $\mathbf{V}_{\mathbf{1}}, \mathbf{V}_{\mathbf{2}}$ and $\mathbf{V}_{\mathbf{3}}$ with $\left\|\mathbf{V}_{\mathbf{1}}\right\| \quad\left\|\mathbf{V}_{\mathbf{2}}\right\| \quad\left\|\mathbf{V}_{\mathbf{3}}\right\|$; Fig. 1 ). To this end, we have built a method based on the SLD fabric tensor to construct a linear and a quadratic fabric tensor that both integrate all three principal axes of individual ellipsoid orientations and lengths.

More generally, two categories of approaches have been developed to treat orientation data, either by analysing vectors (or axes) or by constructing and analysing second-rank tensors. Two types of tensors 


\section{(a) Axis analysis with Fisher statistics}

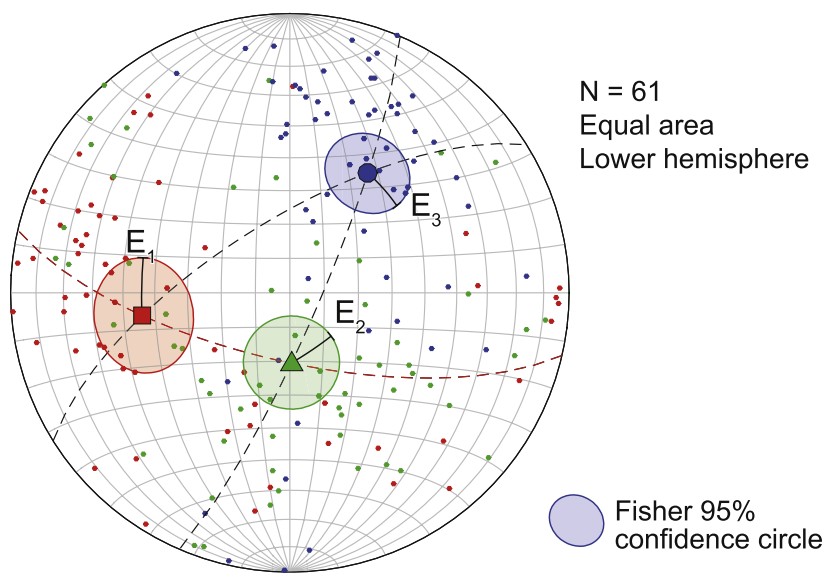

\section{(b) Fabric tensor analysis with Hext-Jelínek}

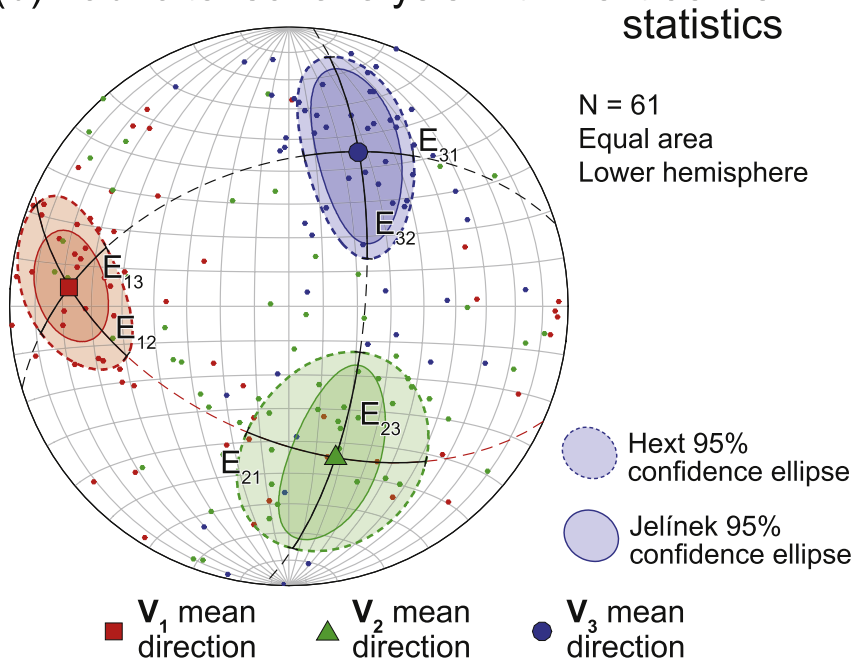

Fig. 2. Mean orientation statistics for the three principal axes defined by a set of ellipsoids. (a) Axis analysis and associated Fisher statistics. $E_{1}, E_{2}$ and $E_{3}$ are cone half-opening angles for $\mathbf{V}_{\mathbf{1}}, \mathbf{V}_{\mathbf{2}}$ and $\mathbf{V}_{\mathbf{3}}$ mean directions. (b) Fabric tensor analysis (linear fabric tensor) with Hext and Jelínek statistics. Hext-Jelínek confidence estimates result in elliptical confidence cones characterised by two half-opening angles (e.g., $E_{13}$ and $E_{12}$ for $V_{1}$ in the direction of $V_{3}$ and $V_{1}$ in the direction of $\mathbf{V}_{\mathbf{2}}$, respectively). The red dashed line highlights the $\mathbf{V}_{\mathbf{1}}-\mathbf{V}_{\mathbf{2}}$ plane, $\mathrm{i}$. e., the foliation defined by the SPO; the lineation corresponds to the direction of $\mathbf{V}_{\mathbf{1}}$. $N$ : number of ellipsoids used for calculations. (For interpretation of the references to colour in this figure legend, the reader is referred to the Web version of this article.)

can be defined: the orientation tensor, constructed for each of the principal directions using their Cartesian coordinates (directional cosines), and the fabric tensor, here constructed using the three principal directions and length of associated axes. Each of the methods provides quantitative estimations of circular and elliptical confidence cones and fabric developments parameters. The nomenclature used is reported in Table 1.

\subsection{Axis analysis and associated confidence estimates}

An intuitive and easily applicable approach analyses orientation and length of each axis (also referred to as vector analysis; after Borradaile, 2003; Tauxe, 2003) by calculating the length $R$ of the mean axis by:

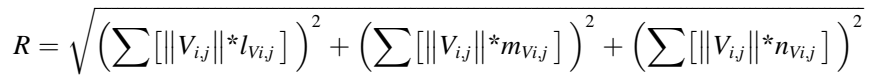

$l_{\mathrm{V} i \mathrm{j}}, m_{\mathrm{V} i \mathrm{j}, \mathrm{j}}$ and $n_{\mathrm{Vi}, \mathrm{j}}$ being the directional cosines of each individual axis and ||$V_{i, j}||$ the length of each individual axis. This method is convenient for statistical estimates of the distribution using Fisher statistics (Fisher, 1953) if data follows a unimodal distribution (one confidence angle; a circular confidence cone; Fig. 2a), and Bingham or Henry - Le Goff statistics (Bingham, 1964; Henry and Le Goff, 1995) if data distribution is bimodal (two confidence angles; an elliptical confidence cone). The main drawback of this approach is that the three principal directions of each ellipsoid are treated independently, whereas the orientation of each principal axis (e.g., $\mathbf{V}_{1}$ ) depends on the orientation of the other two axes $\left(\mathbf{V}_{\mathbf{2}}\right.$ and $\left.\mathbf{V}_{\mathbf{3}}\right)$. The orthogonality of the resulting mean directions will not be preserved and the compiled lineations and foliations defined by an ellipsoid population are without meaning, notably in case of relatively scattered principal axes orientation (Fig. 2a).

\subsection{Orientation tensor}

The mean orientation tensor is a second-rank symmetric tensor constructed using the sum of the directional cosines of a single principal direction (e.g., $\mathbf{V}_{\mathbf{1}}$ ). The method was developed in parallel by Scheidegger (1965) and Watson (1966). The orientation tensor is defined as:

$O_{V i} \quad \sum_{j}^{N} a_{j} a_{j}^{T}$

with $a_{j} \quad\left(\begin{array}{c}l_{j} \\ m_{j} \\ n_{j}\end{array}\right), l, m$, and $n$ being the directional cosines of a specific principal direction (e.g., $\mathbf{V}_{\mathbf{1}}$ ). Thus, $\mathbf{O}_{\mathbf{V i}}$ can be re-written as:

$O_{V i}\left[\begin{array}{ccc}\sum l_{V i, j}^{2} & \sum l_{V i, j} m_{V i, j} & \sum l_{V i, j} n_{V i, j} \\ \sum m_{V i, j} l_{V i, j} & \sum m_{V i, j}^{2} & \sum m_{V i, j} n_{V i, j} \\ \sum n_{V i, j} l_{V i, j} & \sum n_{V i, j} m_{V i, j} & \sum n_{V i, j}^{2}\end{array}\right]$

$\mathbf{O}_{\mathbf{V i}}$ is frequently normalised by the sum of its eigenvalues or by the number of data points. The eigenvectors $\left(\mathbf{v}_{1}, \mathbf{v}_{2}, \mathbf{v}_{3}\right)$ of the greatest, intermediate, and smallest eigenvalues $\left(\lambda_{1}, \lambda_{2}, \lambda_{3}\right)$ of $\mathbf{O}$, corresponds to axes with maximum, intermediate, and minimum strength, respectively. Similar to the axis analysis, $\mathbf{O}_{\mathbf{V i}}$ can only be calculated for an individual direction (e.g., $\mathbf{O}_{\mathbf{v} 1}$ for $\mathbf{V}_{\mathbf{1}}$ axes) but the combination of orientation tensors cannot involve more than 2 principal directions (Lisle, 1989), simply because the sum of $\mathbf{O}$ from the three orthogonal directions is null.

\subsection{Fabric tensor}

Calculation of the three orthogonal principal axes and their interdependent level of confidence alone is not sufficient to fully quantify the fabric tensor because it requires combining information on the three principal axes into a single tensor. This can be done using a fabric tensor that can be described in a linear or quadratic form.

\subsubsection{Linear fabric tensor}

The linear fabric tensor corresponds to the sum of the three orientation tensors of characteristic axes multiplied by their lengths:

$L \quad \sum_{j 1}^{N} V_{1, j} a_{V 1, j} a_{V 1, j}^{T} \quad \sum_{j 1}^{N} V_{2, j} a_{V 2, j} a_{V 2, j}^{T} \quad \sum_{j 1}^{N} V_{3, j} a_{V 3, j} a_{V 3, j}^{T}$

with $a_{V 1, j} \quad\left(\begin{array}{c}l_{V 1, j} \\ m_{V 1, j} \\ n_{V 1, j}\end{array}\right), l, m$, and $n$ being the directional cosines of a specific principal direction (here $\mathbf{V}_{\mathbf{1}}$ ) and $\left\|\mathbf{V}_{\mathbf{1}, j}\right\|$ its length or norm. For example, the first element of equation (4) can be also written as: 


$$
\sum_{j 1}^{N} V_{1, j} a_{V 1, j} a_{V 1, j}^{T}\left[\begin{array}{ccc}
\sum V_{1, j} l_{V 1, j}^{2} & \sum V_{1, j} l_{V 1, j} m_{V 1, j} & \sum V_{1, j} l_{V 1, j} n_{V 1, j} \\
\sum V_{1, j} m_{V 1, j} l_{V 1, j} & \sum V_{1, j} m_{V 1, j}^{2} & \sum V_{1, j} m_{V 1, j} n_{V 1, j} \\
\sum V_{1, j} n_{V 1, j} l_{V 1, j} & \sum V_{1, j} n_{V 1, j} m_{V 1, j} & \sum V_{1, j} n_{V 1, j}^{2}
\end{array}\right]
$$

\subsubsection{Quadratic fabric tensor}

The quadratic fabric tensor design is identical to the fabric tensor based on the SLD method (Ketcham, 2005a; Odgaard et al., 1997; Smit and Odgaard, 1998), but it adopts only the three characteristic axes of individual ellipsoids:

$Q \quad \sum_{j 1}^{N} a_{V 1, j} a_{V 1, j}^{T} \quad \sum_{j 1}^{N} a_{V 2, j} a_{V 2, j}^{T} \quad \sum_{j 1}^{N} a_{V 3, j} a_{V 3, j}^{T}$

with $a_{V 1, j} \quad\left(\begin{array}{cc}V_{1, j} & l_{V 1, j} \\ V_{1, j} & m_{V 1, j} \\ V_{1, j} & n_{V 1, j}\end{array}\right), l, m$, and $n$ being the directional cosines of a specific principal direction (here $\mathbf{V}_{\mathbf{1}}$ ) and $\left\|\mathbf{V}_{\mathbf{1}, \mathbf{j}}\right\|$ its length. For example, the first element of $\mathbf{Q}$, which is the fabric tensor of $\mathbf{V}_{\mathbf{1}}$, can also be written as:

$$
\sum_{j 1}^{N} a_{V 1, j} a_{V 1, j}^{T}\left[\begin{array}{ccc}
\sum V_{1, j}{ }^{2} l_{V 1, j} & \sum V_{1, j}{ }^{2} l_{V 1, j} m_{V 1, j} & \sum V_{1, j}{ }^{2} l_{V 1, j} n_{V 1, j} \\
\sum V_{1, j}{ }^{2} m_{V 1, j} l_{V 1, j} & \sum V_{1, j}{ }^{2} m_{V 1, j}^{2} & \sum V_{1, j}{ }^{2} m_{V 1, j} n_{V 1, j} \\
\sum V_{1, j}{ }^{2} n_{V 1, j} l_{V 1, j} & \sum V_{1, j}{ }^{2} n_{V 1, j} m_{V 1, j} & \sum V_{1, j}{ }^{2} n_{V 1, j}^{2}
\end{array}\right]
$$

of confidence angles that define an elliptical cone around each mean principal axis by integrating the mutually dependant distribution of the individual axes.

First, the Hext method (Hext, 1963; summarised in Tauxe, 2003) uses the mean value of the tensor diagonal elements and uses its deviation to calculate confidence angles. Three angles of confidence of the three mean directions are calculated: $E_{12} \quad E_{21} ; E_{13} \quad E_{31}$; and $E_{23} \quad E_{32}$, with $E_{12}$ being the confidence angle around $\mathbf{V}_{\mathbf{1}}$ in the direction of $\mathbf{V}_{\mathbf{2}}$ (Fig. 2b), usually calculated at a $95 \%$ level of confidence.

The method of Jelínek (Jelínek, 1978; summarised in Tauxe, 2003) requires a larger number of calculations with the use of the covariance matrix of a reassembled tensor to construct three matrices whose eigenvalues are used to derive six different confidence angles: $E_{12}, E_{21}$, $\mathrm{E}_{13}, \mathrm{E}_{31}, \mathrm{E}_{23}$ and $\mathrm{E}_{32}$. Both methods usually provide similar confidence angles, although the Hext method tends to generate larger confidence angles than those produced by the Jelínek method (Fig. 2b; Borradaile, 2003; Tauxe, 2003; Werner, 1997). Both Hext and Jelínek methods require measurements that have uncertainties with zero mean as well as

\subsubsection{Fabric tensor analysis}

Both the linear and the quadratic fabric tensors can be decomposed into eigenvectors $\left(\mathbf{v}_{\mathbf{1}}, \mathbf{v}_{\mathbf{2}}, \mathbf{v}_{\mathbf{3}}\right)$ of the greatest, intermediate, and smallest eigenvalues $\left(\lambda_{1}, \lambda_{2}, \lambda_{3}\right)$, which give the maximum, intermediate, and minimum values of the "moment of inertia" i.e., the mean principal directions and lengths of $\mathbf{V}_{\mathbf{1}}, \mathbf{V}_{\mathbf{2}}$ and $\mathbf{V}_{\mathbf{3}}$ of the fabric ellipsoid, which will be orthogonal (Fig. 2b). From the principal directions, the coordinates of the foliation and the lineation defined by the SPO of the ellipsoids can be calculated and correspond to the $\mathbf{V}_{\mathbf{1}}-\mathbf{V}_{\mathbf{2}}$ plane and to the $\mathbf{V}_{\mathbf{1}}$ axis, respectively.

Several operations are applicable when constructing the orientation tensor. One consists of constructing the fabric tensor with normalised individual ellipsoid size (e.g., by the root of the sum of the square axis length or by the length of the $\mathbf{V}_{\mathbf{1}}$ axis) so that all ellipsoids are weighted equally. This would avoid that the fabric tensor is dominated by a small number of highly anisotropic and large ellipsoids, which potentially form a secondary and weaker fabric (sub-fabric; see discussion below and in the work of Borradaile, 2001). For convenience, both $\mathbf{L}$ and $\mathbf{Q}$ can be normalised by the sum of its eigenvalues.

\subsubsection{Confidence estimates}

Calculations to estimate the data dispersion from second-rank tensors were initially proposed for anisotropy of magnetic susceptibility (AMS) measurements but are applicable to other second-rank tensors on the condition that they are symmetric. The methods permit calculation being normally distributed and small (see discussion in Tauxe, 2003). This can be verified using quantile-quantile plots (Q-Q plots; Tauxe, 2003). A third method was therefore developed by Constable and Tauxe (1990) using a bootstrapping approach that would be preferred in the presence of a sub-fabric (bimodal orientation distribution).

\subsection{Fabric parameters}

Second-order tensors are particularly appropriate to derive quantitative parameters characterising fabrics, as commonly done with structural data, EBSD or AMS. Indeed, fabric analysis can be performed either by using the eigenvalues of the orientation tensor of each axis treated separately $\left(\mathbf{O}_{\mathrm{Vi}} ; K\right.$-index; $P, G, R$ values; parameters introduced below), a combination of eigenvalues of multiple orientation tensors $\left(\mathbf{O}_{\mathbf{V} 1}\right.$ and $\mathbf{O}_{\mathbf{v} 3} ; L S$-index) or simply the eigenvalues of the fabric tensor ( $\mathbf{L}$ and $\mathbf{Q}$; shape parameter $T$, corrected anisotropy $P^{\prime}$ ). All parameters rely on the following ellipsoid shape end-members:

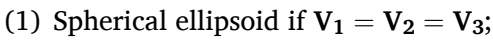

(2) Planar ellipsoid if $\mathbf{V}_{1} \quad \mathbf{V}_{2}>\mathbf{V}_{\mathbf{3}}$;

(3) Linear ellipsoid if $\mathbf{V}_{\mathbf{1}}>\mathbf{V}_{\mathbf{2}} \quad \mathbf{V}_{3}$;

(4) Triaxial (neutral) ellipsoid if $\mathbf{V}_{\mathbf{1}}>\mathbf{V}_{\mathbf{2}}>\mathbf{V}_{\mathbf{3}}$;

(5) The greater the difference between the lengths of each axis, the more anisotropic the ellipsoid becomes.

Most of these parameters can be calculated from a best fit ellipsoid (with $\mathbf{V}_{\mathbf{1}}, \mathbf{V}_{\mathbf{2}}, \mathbf{V}_{\mathbf{3}}$ lengths) to each grain, but also for the fabric tensor 


\section{(a) Ramsay plot (Flinn type)}

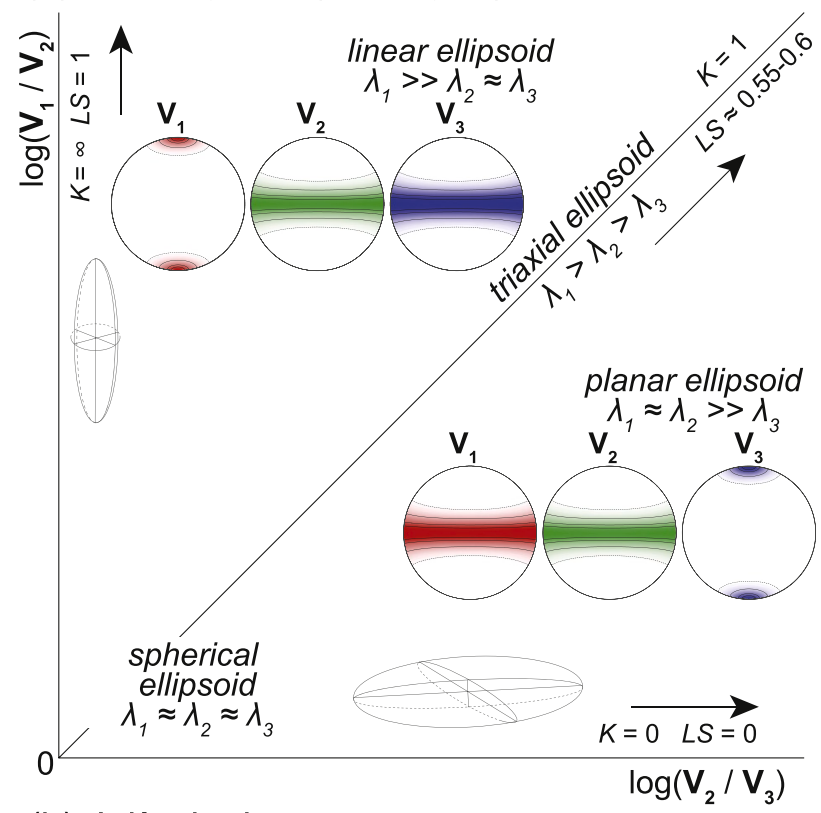

(b) Jelínek plot

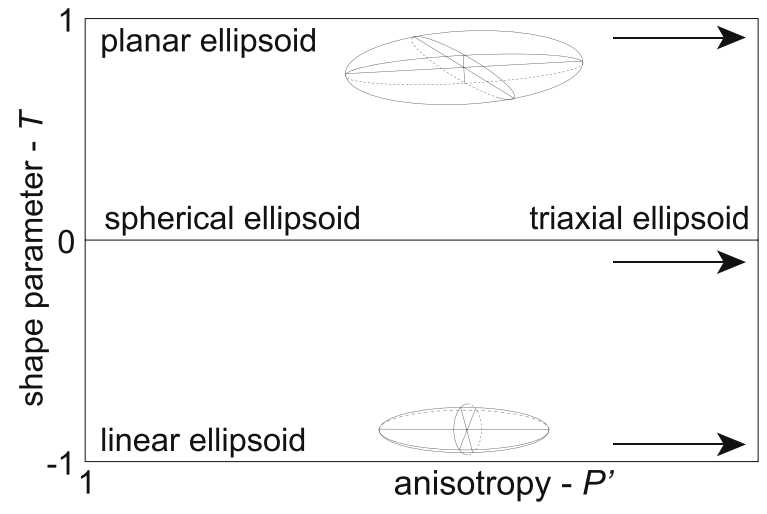

(c) Borradaile-Jackson plot

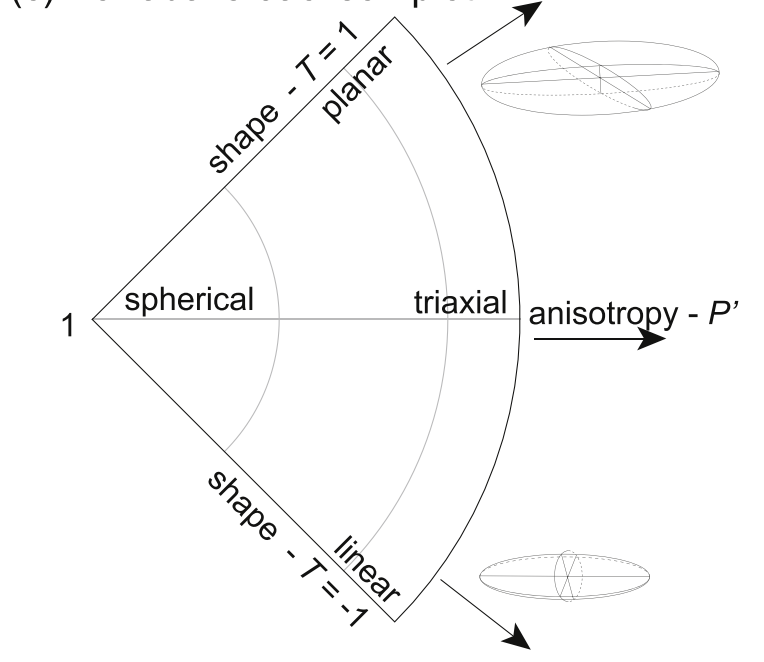

Direction of increasing anisotropy

Fig. 3. Examples of fabric diagrams, associated fabric ellipsoids and ideal distribution of $\mathbf{V}_{\mathbf{1}}, \mathbf{V}_{\mathbf{2}}$ and $\mathbf{V}_{\mathbf{3}}$ orientations. (a) Ramsay plot (modified Flinn diagram; Flinn, 1962; Ramsay, 1967), (b) standard $P^{\prime}-T$ plot (Jelínek plot; Jelínek, 1981) and (c) polar $P^{\prime}$-T plot (Borradaile and Jackson, 2010). Black arrows indicate the direction of increasing degree of anisotropy. eigenvalues $\left(\lambda_{1}, \lambda_{2}, \lambda_{3}\right)$ resulting from the combination of many grains. Various types of diagrams can be used to analyse the parameters calculated from each grain and from the fabric tensor (Fig. 3).

\subsubsection{Shape parameters}

One of the first parameters that was introduced is the $K$-index (Woodcock, 1977), which can be computed using any of the orientation tensors. It is most likely calculated using the orientation tensor of the longest axis of objects (i.e., c-axis for pyroxene, $\mathbf{V}_{\mathbf{1}}$ for ellipsoids) and is defined by Woodcock, 1977 as:

$K \frac{\ln \lambda_{1} / \lambda_{2}}{\ln \lambda_{2} / \lambda_{3}}$

The $K$-index value ranges from 1 to $\infty$ for linear ellipsoids, approaches 1 for spherical and triaxial neutral ellipsoid and ranges from 1 to 0 for planar ellipsoids. It is an analogue to the Flinn (1962) or Ramsay (1967) diagram used in structural geology (Fig. 3a).

Alternatively, Vollmer (1990) proposed the definition of three parameters to estimate the distribution of directions by the calculation of the $P$ (equal 1 for point distribution), $G$ (equal 1 for girdle distribution) and $R$ (equal 1 for random distribution) parameters defined as follows:

$\begin{array}{lllllllll}P & \lambda_{1} & \lambda_{2}, G & 2 & \lambda_{2} & \lambda_{3}, R & 3 & \lambda_{3}\end{array}$

with $P \quad G \quad R \quad 1$ and $\lambda_{1}, \lambda_{2}$, and $\lambda_{3}$ being the three eigenvalues of the orientation tensor of the chosen principal axis.

The $L S$-index of Ulrich and Mainprice (2005) is constructed using the $P, G$, and $R$ parameters of two perpendicular principal directions, commonly the largest and smallest axes (e.g., b- and c-axes of pyroxene, $\mathbf{V}_{\mathbf{1}}$ and $\mathbf{V}_{\mathbf{3}}$ of ellipsoids, respectively), and are defined as:

$L S \frac{1}{2} 2 \quad \frac{P_{V 3}}{G_{V 3} \quad P_{V 3}} \quad \frac{G_{V 1}}{G_{V 1}} P_{V 1}$

with $P_{V 3}$ and $G_{V 3}$ being the $P$ and $G$ parameters calculated using the $\mathbf{V}_{3}$ orientation tensor and $P_{V 1}$ and $G_{V 1}$ being the $P$ and $G$ parameters calculated using the $\mathbf{V}_{\mathbf{1}}$ orientation tensor. Consequently, the $L S$-index equals 1 for linear fabrics and is 0 for planar objects (Fig. 3a).

The shape parameter $T$, developed for AMS measurements, uses a tensor containing the information of the three orthogonal principal directions (i.e., a fabric tensor). It is defined by Jelínek (1981) as:

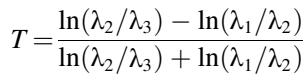

The shape parameter $T$ reflects shape of the fabric ellipsoid: rotational planar (1.0 $T>0.5)$; planar to plano-linear (0.5 $\quad T>0.2)$; plano-linear (0.2 $T>0.2)$; plano-linear to linear $\quad 0.2 \quad T>0.5)$; rotational linear ( $\left.\begin{array}{lll}0.5 & T & 1.0\end{array}\right)$.

\subsubsection{Anisotropy}

The degree of anisotropy of the fabric can be inferred from the "corrected" anisotropy parameter $P^{\prime}$ (Jelínek, 1981) of the fabric tensor, defined as:

$P^{\prime} \quad \exp \sqrt{2} \ln \frac{\lambda_{1}}{\lambda_{m}}{ }^{2} \quad \ln \frac{\lambda_{2}}{\lambda_{m}}{ }^{2} \quad \ln \frac{\lambda_{3}}{\lambda_{m}}{ }^{2}$

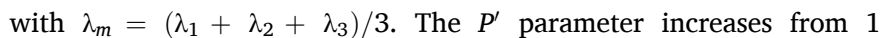
(isotropic) up to values depending on the shape and distribution of its constituents. Both $P^{\prime}$ and $T$ parameters are combined to construct the Jelínek plot, as particularly adapted for rock fabric analysis (Fig. 3b). However, low anisotropy ellipsoids that approach to a spherical shape can be graphically scattered in a standard Jelínek plot. To avoid this graphical bias, an alternative approach was proposed by Borradaile and Jackson (2010) with a polar $P^{\prime}$-T plot (Fig. 3c). 


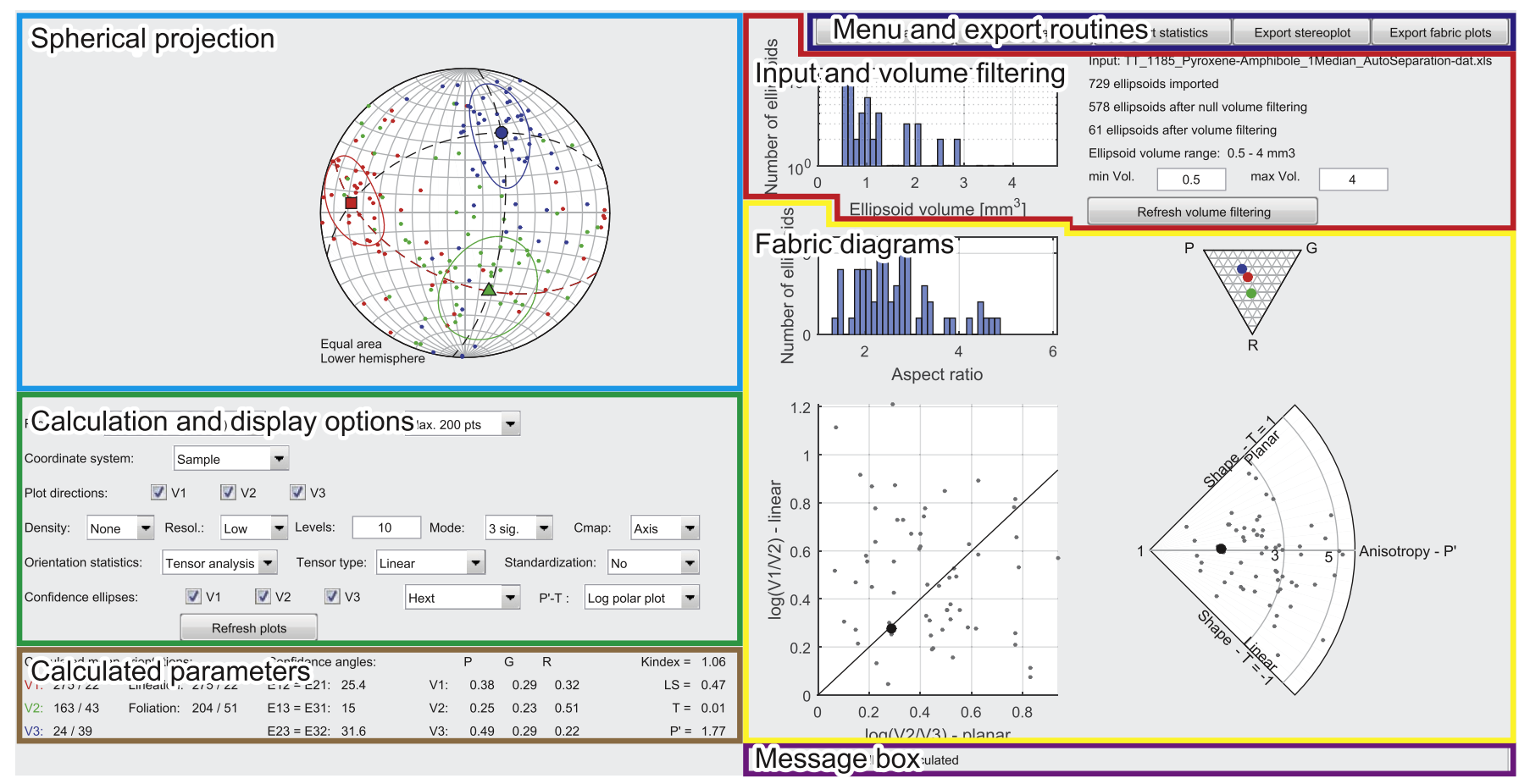

Fig. 4. Graphical user interface of TomoFab allowing to display, analyse and quantify orientation and fabric statistics.

\section{Implementation of the TomoFab program}

The various methods for mean direction calculations, confidence ellipses and parameters informing about the characteristics of the fabric have been incorporated in the TomoFab MATLAB package (a continuously maintained version is available for download at https://github. com/benpetri/tomofab). The code runs on multiple platforms using MATLAB R2015a and newer versions without additional specific toolboxes. The user can run the tomofab.m file, which launches promptly the graphical user interface or GUI (Fig. 4).

The GUI consists of different panels that allow loading and exporting data and plots and then visualizing and filtering input data using the ellipsoid volume. Input files are tab-separated value tables of ellipsoids and sample orientation information. Volume filtering automatically recalculates all statistical parameters and updates all plots. Orientation data are plotted in either equal area (Schmidt) or equal angle (Wülff) nets, however the equal area projection is recommended for fabric analysis to avoid the inherent density distortion of the stereographic equal angle projection. The user can identify data clustering (fabrics and sub-fabrics) using contoured density plots constructed with a modified Kamb method (Vollmer, 1995) displaying densities in $3 \sigma$ (standard deviation) or in multiples of uniform distribution (MUD).

Calculation of mean orientations can be performed either by vector analysis (orthogonality between the mean principal directions is not taken into account) or using the analysis of the fabric tensor (orthogonality between the mean principal directions is preserved). The user has the choice to either use a linear or a quadratic fabric tensor. Depending on the dataset, the user may normalise the size of each ellipsoid by the root of the sum of the squared axis lengths to limit the influence of a small number of large and highly anisotropic ellipsoids on the fabric tensor (the effect of the "sub-fabric" above described). Depending whether the orientation statistics were compiled by vector or tensor analysis, Fisher, Hext and Jelínek confidence cones can be calculated and displayed. Additional statistical methods can also be implemented such as Bingham statistics. For each loop of calculation, orientation parameters (mean $\mathbf{V}_{\mathbf{1}}, \mathbf{V}_{\mathbf{2}}$ and $\mathbf{V}_{\mathbf{3}}$ direction, foliation, and lineation dip directions) are updated at the bottom-left of the window.
Fabric diagrams are updated at each calculation loop and consist of an ellipsoid aspect ratio $\left(\mathbf{V}_{\mathbf{1}} / \mathbf{V}_{\mathbf{3}}\right)$ histogram, a $P G R$ ternary diagram for each principal direction (Vollmer, 1990), a Ramsay plot using a $\log (\|$ $\left.\mathbf{V}_{\mathbf{2}}\|/\| \mathbf{V}_{\mathbf{3}} \|\right)-\log \left(|| \mathbf{V}_{\mathbf{1}} \| /|| \mathbf{V}_{\mathbf{2}}||\right)$ correlation (Fig. 3a; modified from Flinn, 1962; Ramsay, 1967) and a Jelínek plot ( $P^{\prime}-T$; Fig. 3b; Jelínek, 1981) for individual ellipsoid but also for the fabric tensor. Alternatively, a polar $P^{\prime}$-T plot of Borradaile and Jackson (2010) can be used, with either a linear $P^{\prime}$ axis or a $\log \left(P^{\prime}\right)$ axis in case of dataset with scattered individual ellipsoid $P^{\prime}$, which is likely the case with geological material analysed with $\mu$ XCT compared to standard $P^{\prime}$ values derived from AMS dataset (usual $P^{\prime}$ values are ranging between 1 and 1.2).

All calculations can be performed in the sample coordinate system, but the full dataset can be re-oriented back to the geographic coordinate system. To do so, the sample orientation should be known and highlighted by a physical mark before the tomography analysis, in order to apply upside down analysis, strike and dip angle corrections. In such a case, the program reads orientation information and applies corrections for each individual ellipsoid, and performs all calculations (axis or tensor analysis, confidence estimates, fabric parameters) in the geographic coordinate system.

\section{Synthetic case study}

In order to test the different methods of constructing fabric tensors and the implications for confidence ellipses calculation and fabric parameters, we have generated synthetic datasets composed of ellipsoids with variable size, shape, and orientation distribution (Figs. 5-7).

\subsection{Fabric parameters}

The first dataset employed (Figs. 5 and 6) corresponds to a set of 50

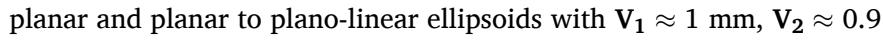
$\mathrm{mm}$ and $\mathbf{V}_{3} \quad 0.6 \mathrm{~mm}$ and 3 linear to plano-linear ellipsoids with $\mathbf{V}_{\mathbf{1}} \quad 2$ $\mathrm{mm}, \mathbf{V}_{\mathbf{2}} \quad 0.9 \mathrm{~mm}$ and $\mathbf{V}_{3} \quad 0.6 \mathrm{~mm}$. These ellipsoids are rotated around their $\mathbf{V}_{\mathbf{1}}$ axis to produce a linear fabric (Figs. $5 \mathrm{a}$ and $6 \mathrm{a}$ ) or rotated around their $\mathbf{V}_{\mathbf{3}}$ axis to produce a planar fabric (Figs. $5 \mathrm{~b}$ and $6 \mathrm{~b}$ ). For better visibility, random noise is added to the orientation of the 
(a) Synthetic linear fabric

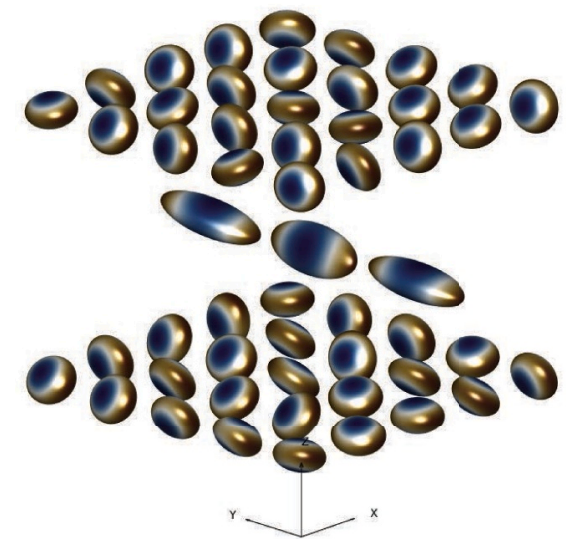

(b) Synthetic planar fabric

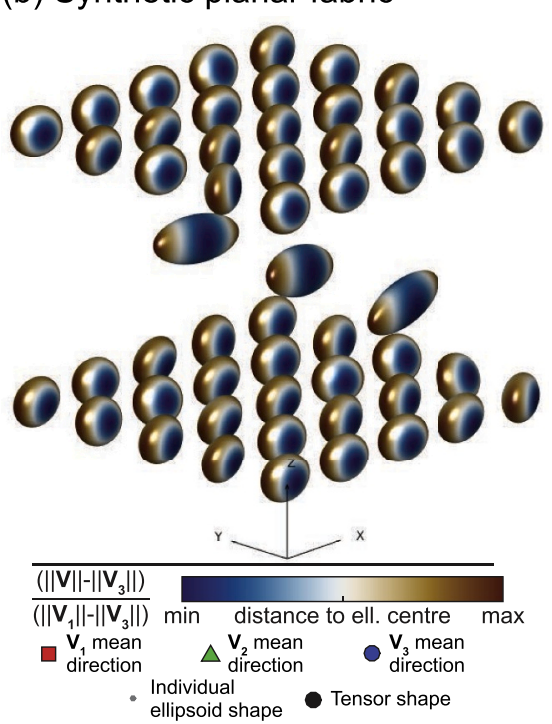

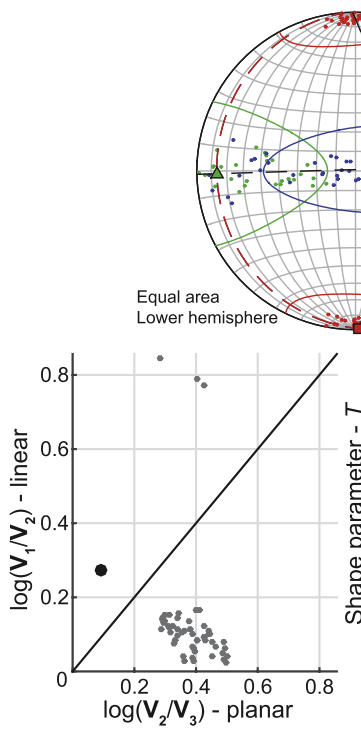
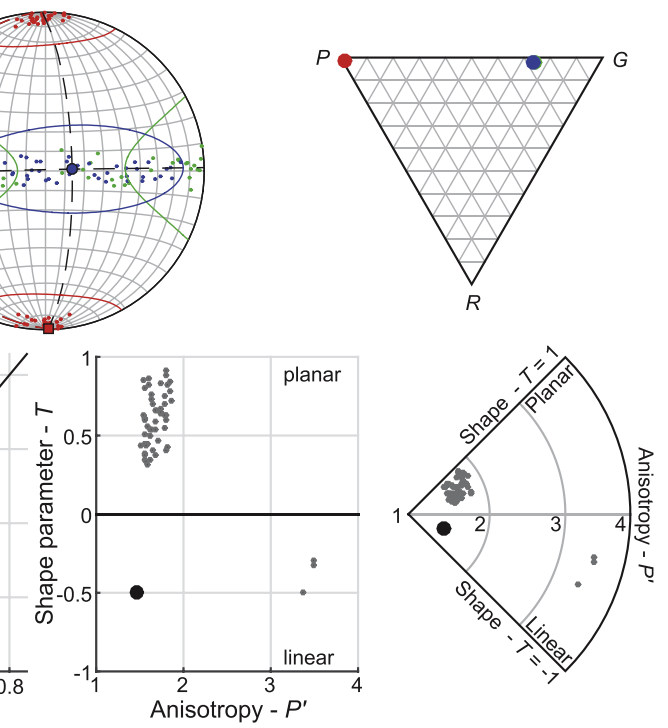

$\left.\mathrm{V}_{2} \mathrm{~N}_{3}\right)$ - plan

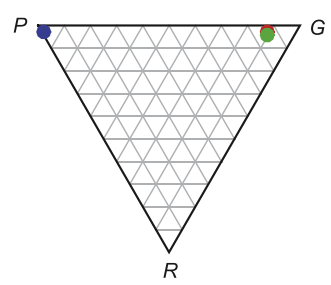

Equal area
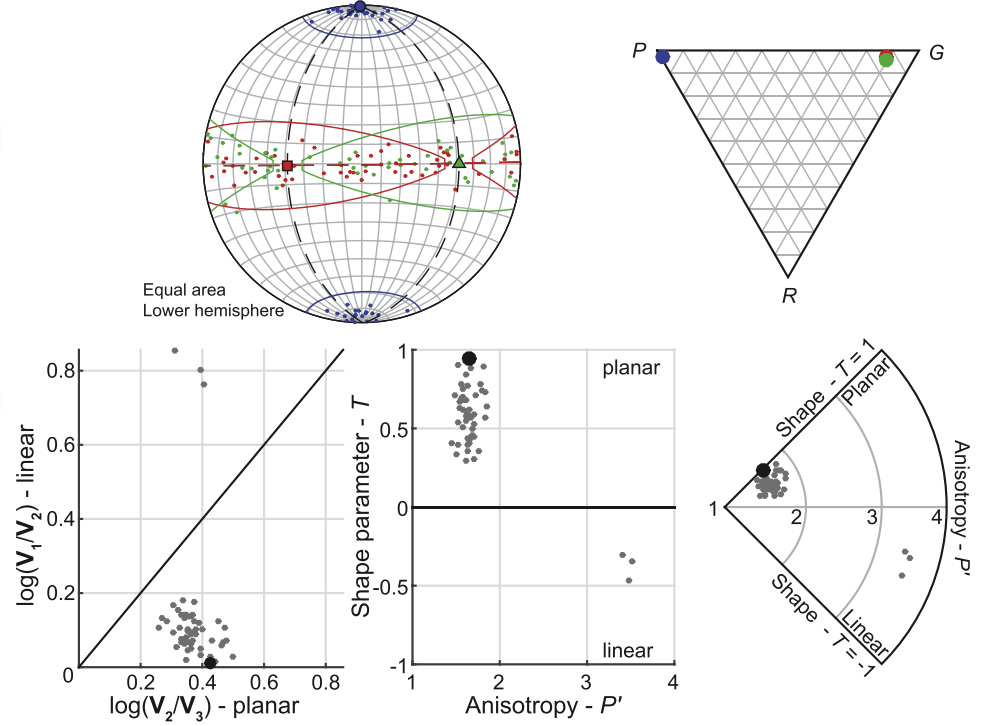

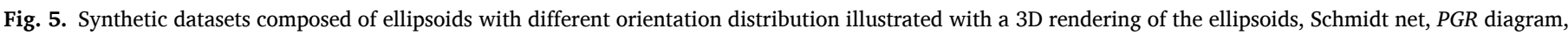

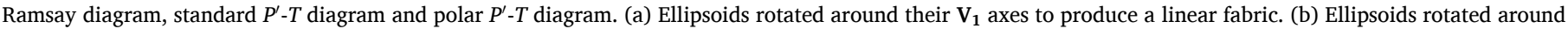

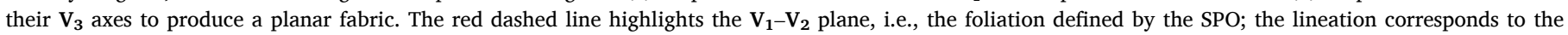

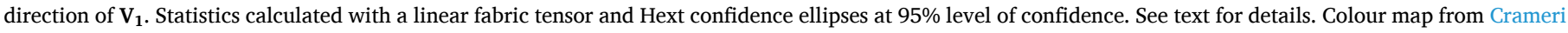

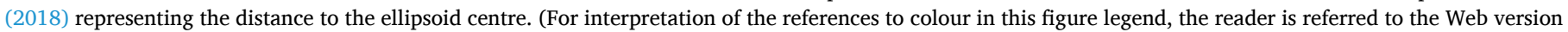
of this article.) 
(a) Synthetic linear fabric
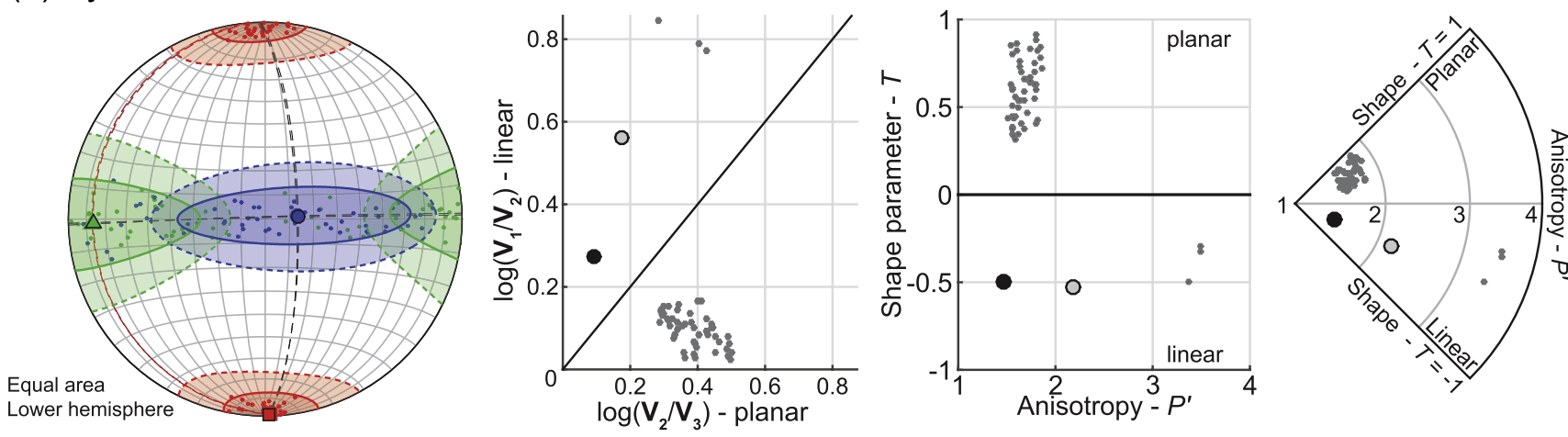

\section{(b) Synthetic planar fabric}
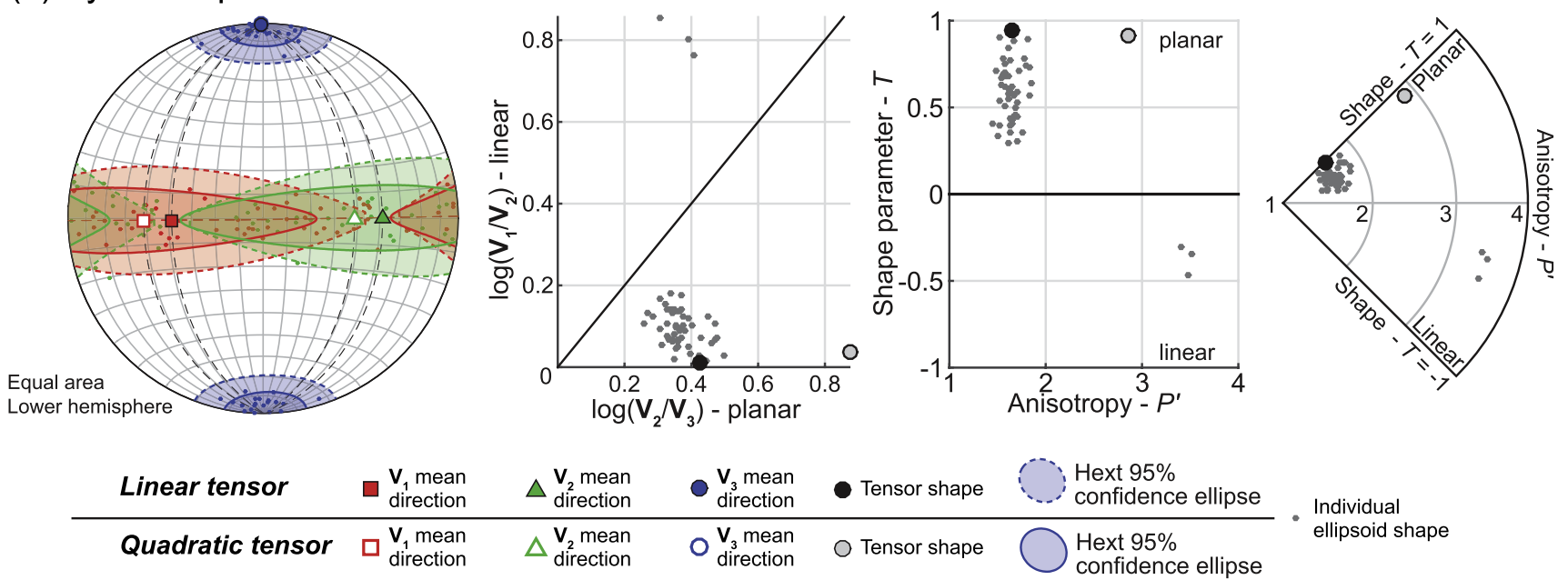

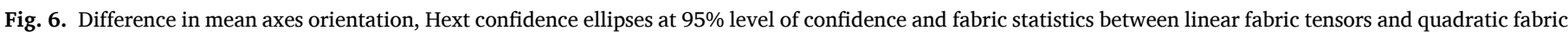

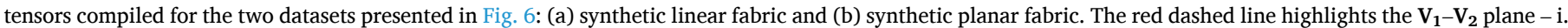

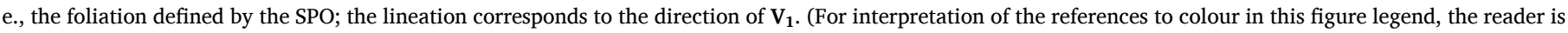
referred to the Web version of this article.)

ellipsoids and the length of their axes.

The synthetic linear fabric (Fig. 5a) displays a point clustering of $\mathbf{V}_{\mathbf{1}}$ axes and girdle distribution of $\mathbf{V}_{\mathbf{2}}$ and $\mathbf{V}_{\mathbf{3}}$ axes, confirmed by the $P G R$ values and diagram. Accordingly, the use of a linear fabric tensor indicates $\mathbf{V}_{\mathbf{1}}$ mean direction at $\mathbf{V}_{\mathbf{1}}$ clustering point, whereas $\mathbf{V}_{\mathbf{2}}$ and $\mathbf{V}_{\mathbf{3}}$ mean directions lie on the girdle defined by $\mathbf{V}_{\mathbf{2}}$ and $\mathbf{V}_{\mathbf{3}}$ individual ellipsoids axes. Compiled Hext confidence angles also highlight the $\mathbf{V}_{2}-\mathbf{V}_{\mathbf{3}}$ girdle distribution, with $\mathrm{E}_{23}$ and $\mathrm{E}_{32}$ angles larger than the other angles. The compiled fabric parameters $\left(K \quad 7.4 ; L S \quad 0.87 ; T \quad 0.50 ; P^{\prime} \quad 1.5\right)$ clearly indicate a linear to plano-linear fabric. It has to be noted that the shape parameter of the fabric ellipsoid has quite high values $\left(\begin{array}{ll}T & 0.5\end{array}\right)$, despite the fact that high $G$ values for $\mathbf{V}_{\mathbf{2}}$ and $\mathbf{V}_{\mathbf{3}}$ would rather lead to lower $T$ values ( $T$ 1). This is the result of the shape of the majority of the individual grains that are planar to plano-linear, avoiding extreme linear $T$ values.

The synthetic planar fabric (Fig. 5b) displays a point clustering of $\mathbf{V}_{\mathbf{3}}$ axes whereas $\mathbf{V}_{\mathbf{1}}$ and $\mathbf{V}_{\mathbf{2}}$ axes are distributed along a girdle, in agreement with the $P G R$ values of the three principal axes. Using a linear fabric tensor, the mean $\mathbf{V}_{\mathbf{1}}$ and $\mathbf{V}_{\mathbf{2}}$ directions lie on the girdle defined by the individual ellipsoid principal axes, whereas mean $\mathbf{V}_{\mathbf{3}}$ is located on the $\mathbf{V}_{\mathbf{3}}$ cluster. The derived Hext confidence angles shows high opening values for $E_{12}$ and $E_{21}$ whereas the other confidence angles are rather small. In agreement to this, the fabric parameters indicate a clearly planar fabric ( $\left.K \quad 0.06 ; L S \quad 0.06 ; T \quad 0.95 ; P^{\prime} \quad 1.6\right)$. The shape parameter $(T)$ is quite high in comparison to the one compiled with the synthetic linear fabric (Fig. 5a) and is explained by the planar and planar to plano-linear shape of individual ellipsoids. This clearly demonstrates the influence of the shape of individual ellipsoids on the resulting fabric ellipsoid.

\subsection{Linear vs. quadratic fabric tensor}

Using the same two synthetic datasets, we have compiled mean orientation, confidence angles and fabric parameters from linear fabric tensors and quadratic fabric tensors (Fig. 6). The calculated mean principal axes for the synthetic linear fabric is almost identical between the two tensors. However, the mean principal directions of $\mathbf{V}_{\mathbf{1}}$ and $\mathbf{V}_{\mathbf{2}}$ for the synthetic planar fabric shows misorientation of $\sim 10$ but remains on the girdle defined by the $\mathbf{V}_{\mathbf{1}}$ and $\mathbf{V}_{\mathbf{2}}$ individual ellipsoid orientations. The Hext confidence angles calculated using the two methods present systematically smaller opening angles with a quadratic fabric tensor than with a linear fabric tensor. The fabric parameters that are derived from both linear (Fig. $6 \mathrm{a} ; K \quad 7.4 ; L S \quad 0.87 ; T \quad 0.53 ; P^{\prime} \quad 2.2$ ) and planar synthetic fabrics (Fig. 6 b; $K \quad 0.06 ; L S \quad 0.06 ; T \quad 0.92 ; P^{\prime} \quad 2.9$ ) still indicates a plano-linear to linear fabric and a planar fabric, respectively. $K$ - and $L S$ - indexes are identical as they are compiled with $\mathbf{V}_{\mathbf{1}}$ and $\mathbf{V}_{\mathbf{3}}$ orientation tensors. This is not the case with the parameters derived from fabric tensors, notably for the anisotropy parameter $P^{\prime}$ : the squared axes length favours long axes more than small axes when constructing 
(a) Non-normalized ellipsoids

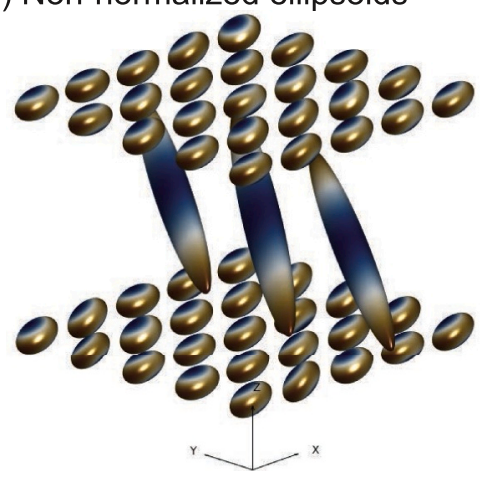

(b) Normalized ellipsoids
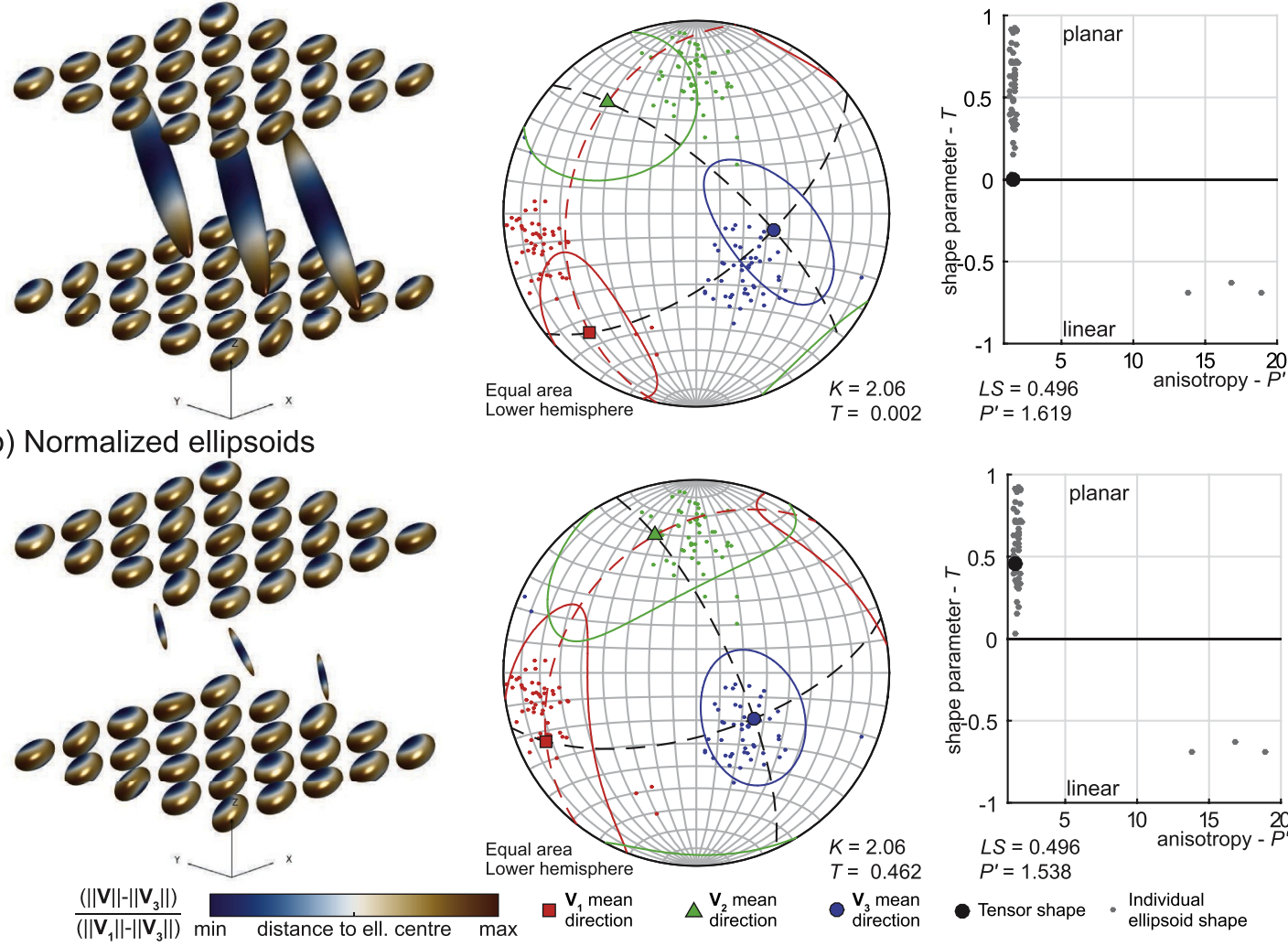

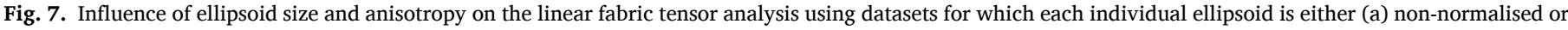

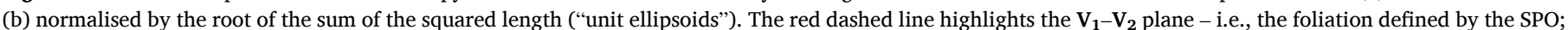

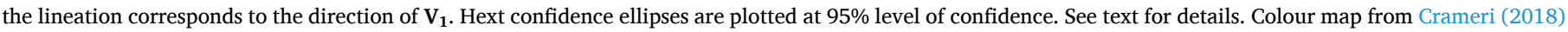

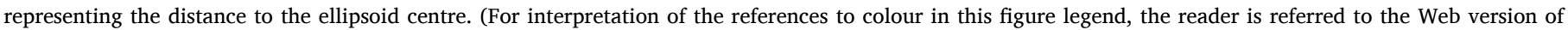
this article.)

the tensor, but also when deriving its eigenvalues and eigenvectors. This has several consequences: (1) the calculated mean principal directions with a quadratic fabric tensor are predominantly influenced by the orientation of the large ellipsoids compared to the small ellipsoids, explaining the misorientation of $\mathbf{V}_{\mathbf{1}}$ and $\mathbf{V}_{\mathbf{2}}$ observed in Fig. $6 \mathrm{~b}$; and (2) the quadratic fabric ellipsoid has a different shape parameter $(T)$ value and higher degree of anisotropy $\left(P^{\prime}\right)$, which cannot be compared to individual ellipsoids.

\subsection{Standardised fabric tensor}

The second dataset employed (Fig. 7) is composed of 50 planar to plano-linear ellipsoids with $\mathbf{V}_{1} \quad 1 \mathrm{~mm}, \mathrm{~V}_{2} \quad 0.9 \mathrm{~mm}$ and $\mathbf{V}_{3} \quad 0.6 \mathrm{~mm}$ and 3 larger linear ellipsoids with $\mathrm{V}_{1} \quad 6 \mathrm{~mm}, \mathrm{~V}_{2} \quad 0.9 \mathrm{~mm}$ and $\mathrm{V}_{3}$ $0.6 \mathrm{~mm}$. The axes orientation and length are varied slightly for better visualisation: the three large ellipsoids are defining a $\mathbf{V}_{\mathbf{1}}$ cluster at 205/ 25 whereas the set of small ellipsoids are defining a $\mathbf{V}_{\mathbf{1}}$ cluster at 260/20.

As fabric tensors are constructed using the length of the characteristic axes of ellipsoids, more weight is attributed to large and highly anisotropic ellipsoids than to small and isotropic ellipsoids. The influence of such outlier ellipsoids is moderate when using linear fabric tensors but dominant using quadratic fabric tensors. This effect is depicted in Fig. 7a, where the mean principal axes are lying in between the two clustering points, in particular for mean $\mathbf{V}_{\mathbf{1}}$ and $\mathbf{V}_{\mathbf{3}}$ axes. Confidence ellipses clearly show a triaxial fabric ellipsoid (note that the exemplified bimodal distribution might not be suitable for Hext and Jelínek confidence estimates; see section 2.3.4.). This is confirmed by the fabric statistics, especially by the $T$ value $(K \quad 2.1 ; L S \quad 0.50 ; T$ $\left.0.00 ; P^{\prime} \quad 1.6\right)$. The mean axes orientation, the confidence ellipses and the fabric statistics indicate that the three large ellipsoids define a subfabric that overcomes the fabric defined by the 50 smaller ellipsoids. This may occasionally lead to a decoupling between indexes derived from orientation tensors and indexes derived from fabric tensors (here $K$ $>1$ and $T \quad 0$ ). The sub-fabric effect is attenuated by normalising each individual ellipsoid by the root of the sum of the squared axes length to obtain "unit ellipsoids" (see details in Borradaile, 2001). In such a case, mean principal directions and fabric statistics are ruled by the most populated set of ellipsoids rather than by the largest and most anisotropic ellipsoids (Fig. 7b), which mean principal directions localised at individual axes clustering points, and confidence angles and fabric statistics approaching values expected from the set of 50 small ellipsoids ( $K$

$$
\left.2.1 ; L S \quad 0.50 ; T \quad 0.46 ; P^{\prime} \quad 1.5\right) \text {. }
$$

\section{Natural case study}

The natural example represents a sub-volume of an oriented core ( $\sim 45$-mm diameter) collected with a portable rock drill (Fig. 8). It consists of a pyroxene-bearing gabbro from the exposed lower-crustal section of the Ivrea-Verbano Zone, in the gabbro-norite zone of the Upper Mafic Complex (sample MC07-02; 45.84826 N; 8.19640 E; Val Mastallone, NW Italy). The sample is composed of pyroxenes, plagioclase, and oxides (magnetite and ilmenite) and presents a welldeveloped foliation and lineation marked by the preferred orientation of magmatic pyroxene grains (Fig. 8a-c). Notably, the study of this sample allows a better understanding of the dynamics and the fabric development related to magma flow and emplacement in the lower 

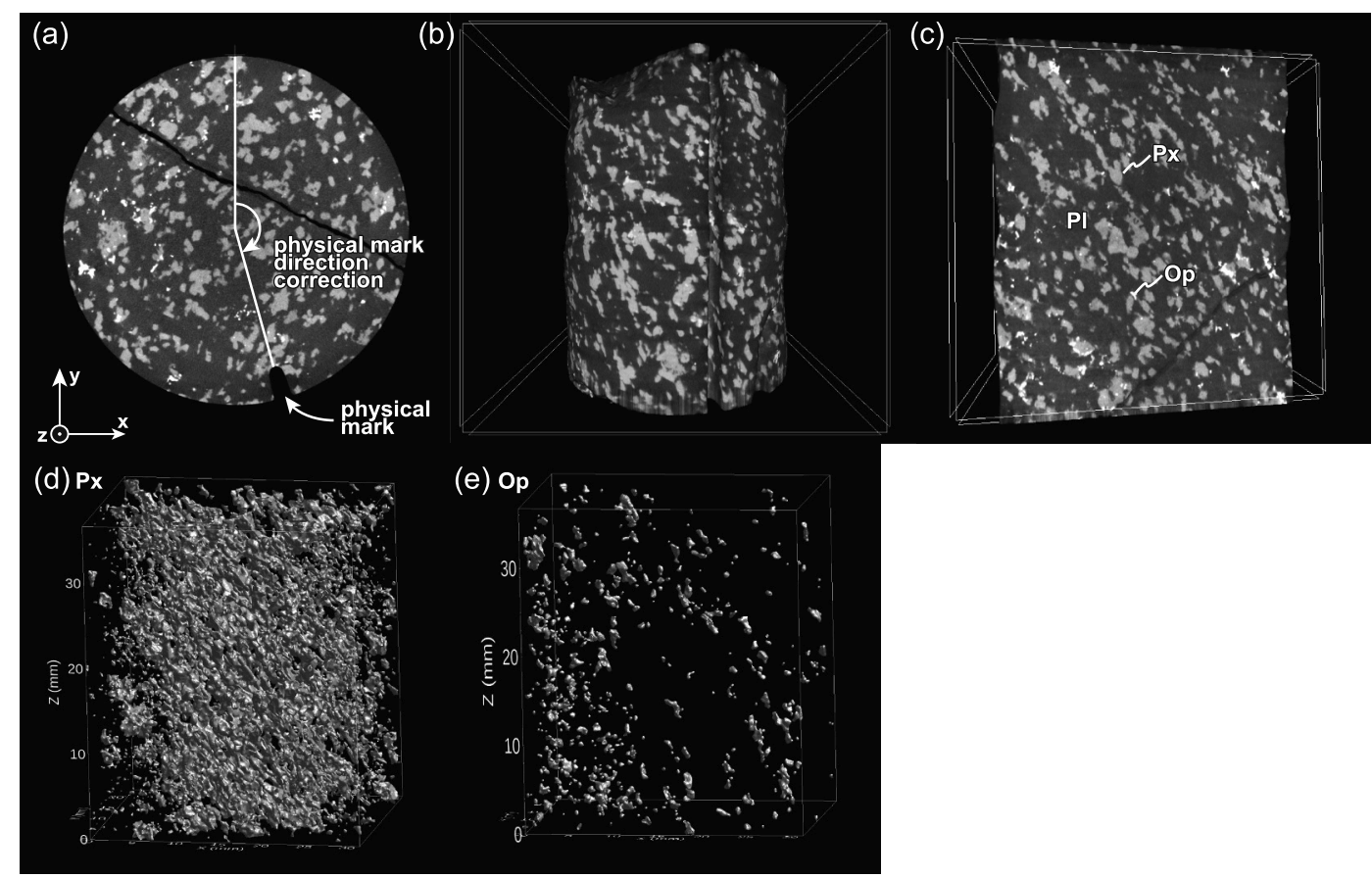

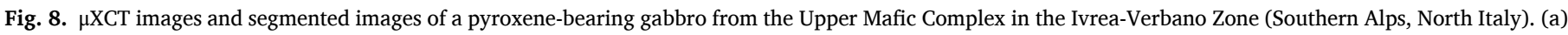

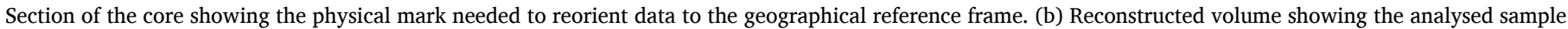

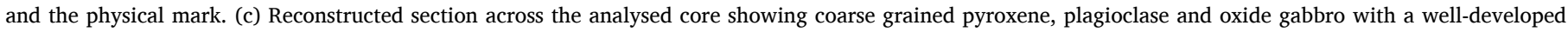

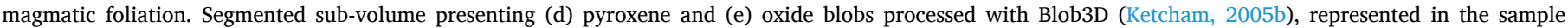
reference frame. Px: pyroxenes; Pl: plagioclase; Op: opaque mineral (oxides; magnetite and ilmenite).

crust.

The sample was imaged with a Bruker SkyScan $1173 \mathrm{X}$-ray microtomographer at the University of Lausanne (Switzerland) using a source voltage of $130 \mathrm{kV}$ and a source current of $61 \mu \mathrm{A}$ for $6 \mathrm{~h}$, producing 801 slices with a $50 \mu \mathrm{m}$ spatial resolution. Image segmentation and both automatic and manual separations (i.e., defining and isolating a set of connected voxels that constitutes a blob; see Appendix A for details) were performed using Blob3D (Ketcham, 2005b) before measuring each blob centre coordinates, volume and best-fit ellipsoid axis length and orientation using the same software. Figs. 9-10 present fabric and orientation distribution of pyroxene and oxide grains that were manually separated and selected by their volume $\left(0.5-8.5 \mathrm{~mm}^{3}\right.$ for pyroxene; $0.1-2.4 \mathrm{~mm}^{3}$ for oxide). A comparison between automatic and manual segmentation results is reported in Table 2 and as Supplementary Material (see Appendix A). In order to compare the results of our approach to state-of-the-art methods, we performed a Star Length Distribution (SLD) analysis using Quant3D (Ketcham, 2005a; Odgaard et al., 1997; Smit and Odgaard, 1998) on the same set of images segmented with Blob3D. The SLD is a continuum method of fabric characterization that does not require a separation of grains or even to consider grains, but suffers from being grain size sensitive; a large grain will be sampled and integrated several times, whereas smaller grains may even not be integrated.

Individual best-fit ellipsoid of both pyroxene and oxide grains are scattered but pyroxene grains are preferentially plano-linear, whereas oxide grains are more elongated, being preferentially linear to planolinear (Fig. 9). In the absence of solid-state deformation, these individual grain shape parameters correspond to the almost ideal shape of magmatic pyroxenes and oxides.

Orientation of individual grains best-fit ellipsoid of pyroxene grains (Fig. 10a) shows a clear $\mathbf{V}_{\mathbf{1}}$ vertical point distribution with a weak vertical girdle that is striking towards the NNE. Conversely, $\mathbf{V}_{3}$ distribution presents a horizontal girdle distribution with ESE and WNW point maxima. The analysis of the linear fabric tensor compiled with the same dataset constrains a vertical foliation and a vertical lineation at $\sim 10$ from the field measured foliation and lineation. The fabric defined by the pyroxene grains is linear to plano-linear as indicated by the analysis of the orientation tensors $(K \quad 1.776 ; L S \quad 0.625)$ and the linear fabric tensor ( $T \quad 0.248 ; P^{\prime} \quad$ 1.537). The SLD analysis of segmented but not separated images indicate a plano-linear fabric (Fig. 9a; $\lambda_{1}$ $0.384 ; \lambda_{2} \quad 0.335 ; \lambda_{3} \quad 0.281$; equivalent to $T \quad 0.117$ and $P^{\prime} \quad 1.367$ ) with higher equivalent $T$ values than those ones derived from the linear fabric tensor, likely due to the integration of grains aggregates when using the SLD method.

The orientation distribution of oxide grains (Fig. 10b) is moderately scattered. The distribution of $\mathrm{V}_{\mathbf{1}}$ orientations draws a moderately defined girdle that steeply dips to the ESE from which a vertical point distribution can be noticed. On the other hand, $\mathbf{V}_{\mathbf{3}}$ distribution defines two ESE and WNW horizontal, but scattered, point distribution. The analysis of the linear fabric tensor presents rather similar orientations to those derived for the pyroxene grains, but with larger Hext and Jelínek confidence estimates due to the scattered distribution of individual grain orientation. Although oxide grains are individually quite elongated (Fig. 9b), the fabric they define is planar to plano-linear $\left(\begin{array}{ll}K & 0.653 ; L S\end{array}\right.$

$\left.0.260 ; T \quad 0.302 ; P^{\prime} \quad 1.323\right)$. The results of both orientation and fabric tensor analysis are in good agreement with the SLD analysis (Fig. 9b; $\lambda_{1} \quad 0.372 ; \lambda_{2} \quad 0.342 ; \lambda_{3} \quad 0.286$; equivalent to $T \quad 0.351$ and $P^{\prime}$ 1.307).

In general, the use of automatically separated grains allows to give a first order constraint on the grain shape and fabric properties, but the analysis is disturbed by the integration of a large number of grain aggregates as well as randomly oriented, very small grains that are ruled out by volume filtering using TomoFab (see comparison in Appendix A). We have to highlight that in our example, both automatically and manually separated datasets lead to similar fabric properties which may not be the case for all datasets, notably in case of interconnected pores or grains (aggregates). However, manual separation clearly increases the quality of the dataset, allowing to better document the difference in fabric properties between the pyroxene and the oxide grain population that relates different stress regime during the sequential crystallisation 
(a) Pyroxene $(N=538)$

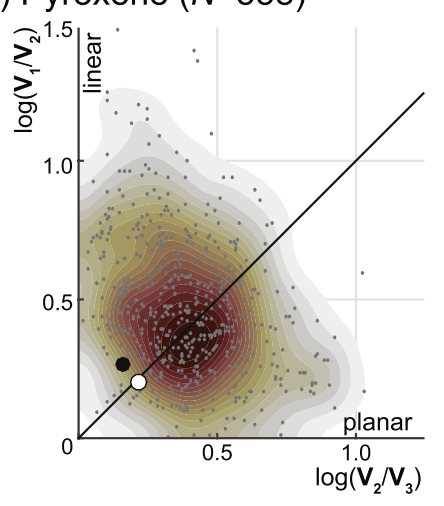

(b) Oxide $(N=306)$

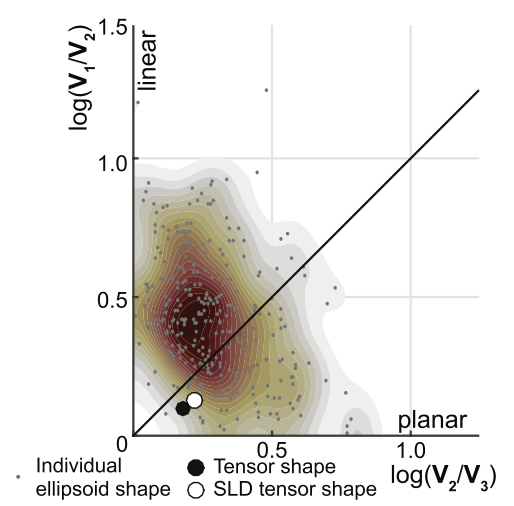

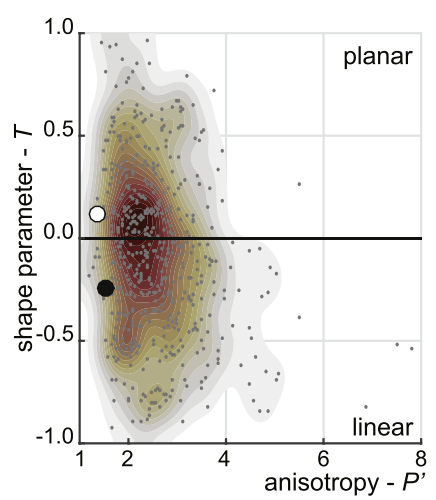

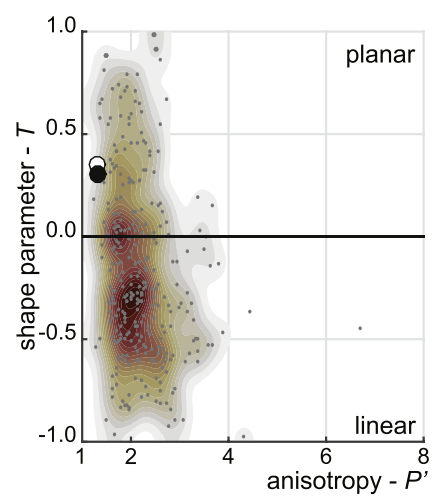

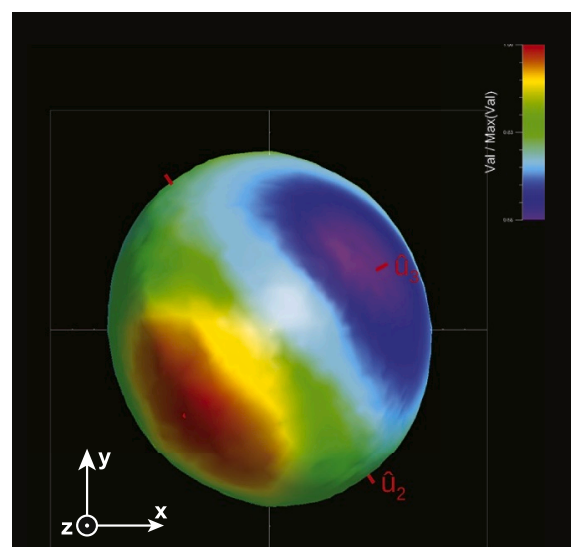

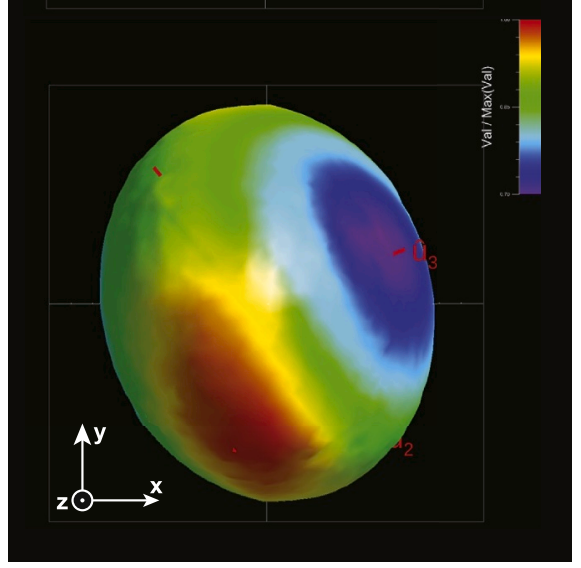

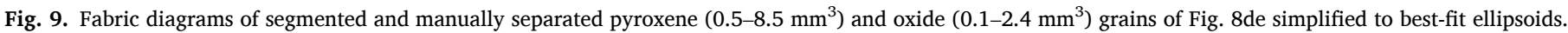

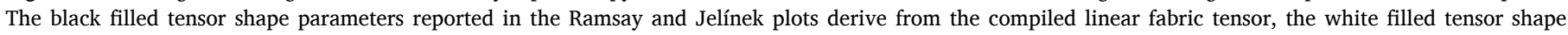

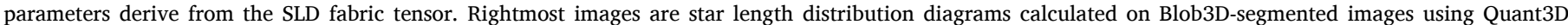

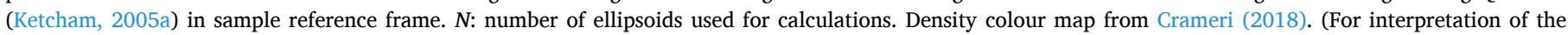
references to colour in this figure legend, the reader is referred to the Web version of this article.) 

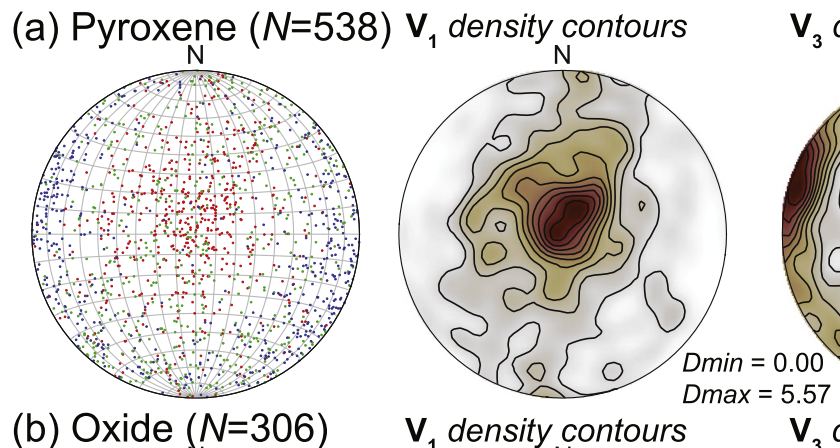

$\mathbf{V}_{3}$ density contours
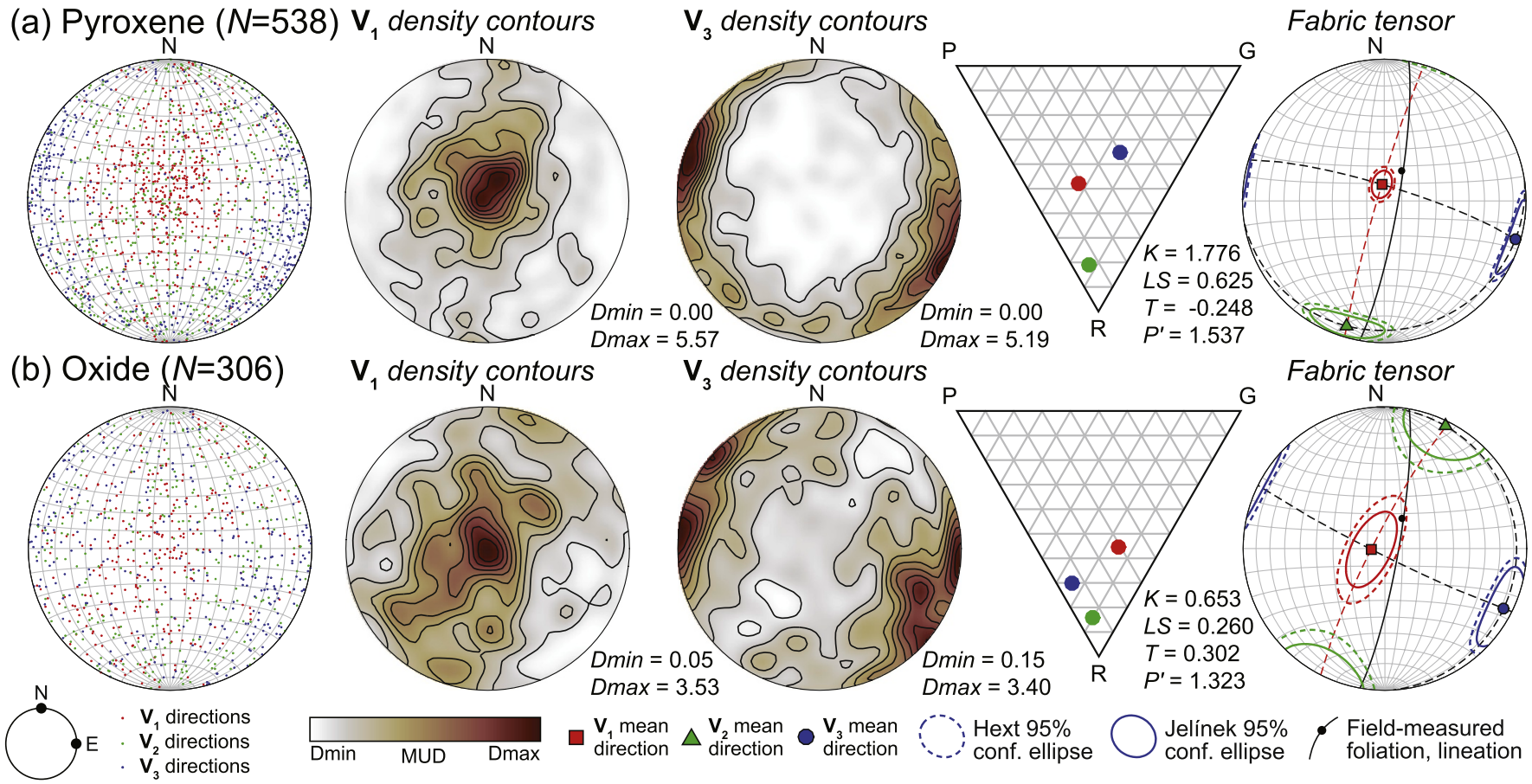

\begin{abstract}
(1)
\end{abstract}
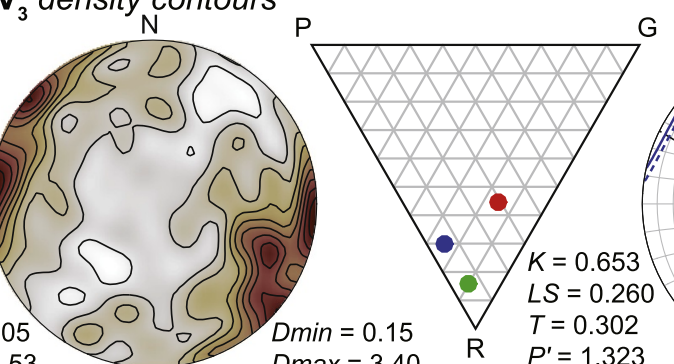

Fabric tensor

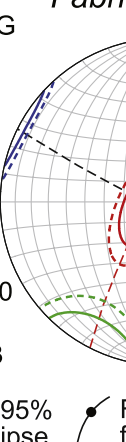

R $\quad P^{\prime}=1.323$ $\mathrm{N}$ $\mathrm{N}$

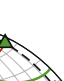

$\mathbf{v}_{2}$ mean
direction $\begin{aligned} & \mathbf{v}_{3} \text { mean } \\ & \text { direction }\end{aligned}$ Jelínek $95 \%$
conf. ellipse foliation, lineation

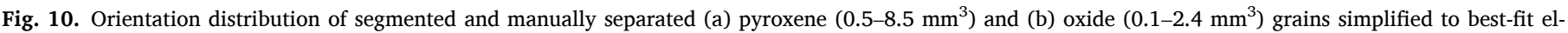

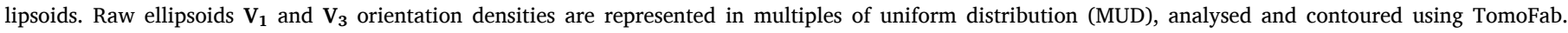

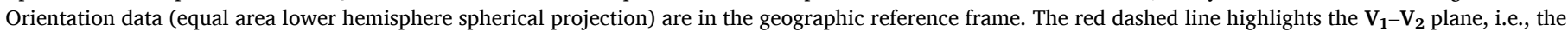

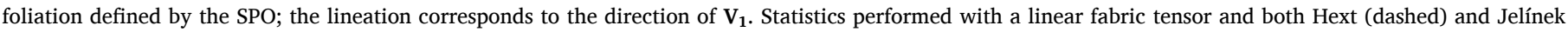

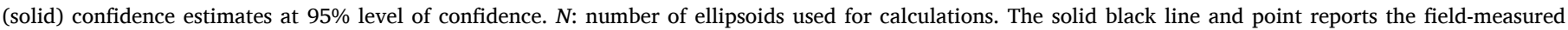

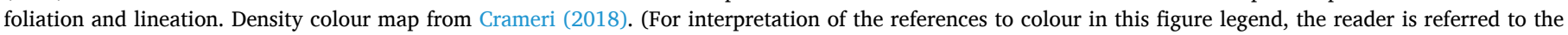
Web version of this article.)

Table 2

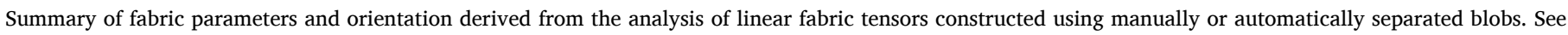
Figs. 9-10 and Appendix A for related diagrams.

\begin{tabular}{|c|c|c|c|c|c|c|c|c|}
\hline & Separation & $N$ & $K$ & $L S$ & $T$ & $P^{\prime}$ & Foliation & Lineation \\
\hline \multirow[t]{2}{*}{ Pyroxene } & manual & 538 & 1.776 & 0.625 & 0.248 & 1.537 & $286 / 86$ & $352 / 80$ \\
\hline & automatic & 658 & 1.209 & 0.526 & 0.103 & 1.468 & $287 / 86$ & $342 / 83$ \\
\hline \multirow[t]{2}{*}{ Oxide } & manual & 306 & 0.653 & 0.260 & 0.302 & 1.323 & $297 / 83$ & $267 / 82$ \\
\hline & automatic & 260 & 0.798 & 0.275 & 0.108 & 1.400 & $301 / 82$ & $301 / 82$ \\
\hline
\end{tabular}

of the various mineralogical phases (Table 2). Notably, the oxide grain population, although being linear to plano-linear in general, the fabric they define is planar to plano-linear showing that they behave as passive markers of the deformation - i.e., individual grain shape does not always translate deformation regimes.

\section{Conclusion}

We have designed and constructed a versatile fabric tensor analysis tool to characterise rock fabrics using segmented micro-computed tomography datasets. We defined the linear fabric tensor and the quadratic fabric tensor using the sum of the orientation tensors of the three characteristic axes of grains (simplified to best fit ellipsoids) multiplied by the length or squared length of each axis, respectively. The use of the fabric tensor allows calculation of three orthogonal mean principal axes, their elliptical cones of confidence and the two main parameters relevant to rock fabric quantitative characterisation: the fabric ellipsoid shape and the degree of anisotropy. This approach is implemented in TomoFab, an open-source MATLAB package that is freely available for download. The method permits quantitative comparison of rock fabrics of different samples imaged by micro-computed tomography, but also to fabrics constrained by other independent methods (EBSD, AMS, and seismic anisotropy). The examples described above demonstrate the wide applicability of the method. Ultimately, the software can be more broadly used in Earth and material sciences and, potentially, in other fields when considering analyses of material fabrics and porosity.

\section{Computer code availability}

TomoFab is an open source MATLAB package available for download at https://github.com/benpetri/tomofab. TomoFab is developed under a GNU licence by Benoît PETRI (see corresponding author information). The code runs on multiple platforms using MATLAB R2015a and newer versions without additional specific toolboxes. 


\section{Declaration of competing interest}

The authors declare that they have no known competing financial interests or personal relationships that could have appeared to influence the work reported in this paper.

\section{Acknowledgments}

This work was supported by the University of Lausanne and the University of Strasbourg and by an ExxonMobil research grant to BP. BA received funding for this project from the Swedish Research Council grant 2018-03414. MP acknowledges the Ambizione Fellowship (PZOOP2_168166), supported by the Swiss National Science Foundation, for fieldwork and rock sampling in the Ivrea-Verbano Zone (Italy) and rock analysis by $\mu \mathrm{XCT}$ at UNIL. Pauline Le Maire is thanked for fruitful discussions around orientation data treatment and MATLAB programming. Lukas P. Baumgartner and Benita Putlitz are gratefully thanked for giving access to the UNIL $\mu$ XCT facility as well as Othmar Müntener for insightful discussions. We thank Frederick W. Vollmer, the two anonymous reviewers, as well as the Editor-in-chief Dario Grana and the Associate Editor Eileen Martin for their helpful comments and editorial handling.

\section{Appendix A. Supplementary data}

Supplementary data to this article can be found online at https://doi. org/10.1016/j.cageo.2020.104444.

\section{References}

Bascou, J., Barruol, G., Vauchez, A., Mainprice, D., Egyrio-Silva, M., 2001. EBSDmeasured lattice-preferred orientations and seismic properties of eclogites. Tectonophysics 342, 61-80.

Benn, D.I., 1994. Fabric shape and the interpretation of sedimentary fabric data. SEPM J. Sediment. Res. 64A https://doi.org/10.1306/D4267F05-2B26-11D7 8648000102C1865D.

Benn, K., Allard, B., 1989. Preferred mineral orientations related to magmatic flow in ophiolite layered gabbros. J. Petrol. 30, 925-946.

Bingham, C., 1964. Distributions on the Sphere and on the Projective Plane. PhD Thesis Yale University.

Borradaile, G.J., 2003. Statistics of Earth Science Data: Their Distribution in Time, Space and Orientation. Springer Science \& Business Media.

Borradaile, G.J., 2001. Magnetic fabrics and petrofabrics: their orientation distributions and anisotropies. J. Struct. Geol. 23, 1581-1596. https://doi.org/10.1016/S01918141(01)00019-0.

Borradaile, G.J., Jackson, J.A., 2010. Structural geology, petrofabrics and magnetic fabrics (AMS, AARM, AIRM). J. Struct. Geol. 32, 1519-1551.

Bryon, D.N., Atherton, M.P., Hunter, R.H., 1995. The interpretation of granitic textures from serial thin sectioning, image analysis and three-dimensional reconstruction. Mineral. Mag. 59, 203-211. https://doi.org/10.1180/minmag.1995.059.395.05.

Chopin, F., Schulmann, K., Stípska, P., Martelat, J.E.E., Pitra, P., Lexa, O., Petri, B., 2012. Microstructural and metamorphic evolution of a high-pressure granitic orthogneiss during continental subduction (Orlica-Snieżnik dome, Bohemian Massif). J. Metamorph. Geol. 30, 347-376. https://doi.org/10.1111/j.1525 1314.2011.00970.x.

Constable, C., Tauxe, L., 1990. The bootstrap for magnetic susceptibility tensors J. Geophys. Res. 95, 8383. https://doi.org/10.1029/JB095iB06p08383.

Crameri, F., 2018. Scientific colour-maps. Zenodo. https://doi.org/10.5281/ zenodo. 1243862

Fisher, N.I., Lewis, T., Embleton, B.J.J., 1993. Statistical Analysis of Spherical Data. Cambridge university press.

Fisher, R., 1953. Dispersion on a sphere. Proc. R. Soc. A Math. Phys. Eng. Sci. 217, 295-305. https://doi.org/10.1098/rspa.1953.0064.

Flinn, D., 1962. On folding during three-dimensional progressive deformation. Q. J. Geol. Soc. 118, 385-428. https://doi.org/10.1144/gsjgs.118.1.0385.

Harrigan, T.P., Mann, R.W., 1984. Characterization of microstructural anisotropy in orthotropic materials using a second rank tensor. J. Mater. Sci. 19, 761-767. https:// doi.org/10.1007/BF00540446.

Heilbronner, R.P., 1992. The autocorrelation function: an image processing tool for fabric analysis. Tectonophysics 212, 351-370. https://doi.org/10.1016/0040-1951 (92) $90300-U$.

Henry, B., Le Goff, M., 1995. Application de l'extension bivariate de la statistique Fisher aux donnees d'anisotropie de susceptibilite magnetique: integration des incertitudes de mesure sur l'orientation des directions principales. Comptes rendus l'Academie des Sci. Serie 2. Sci. la terre des planetes 320, 1037-1042.

Hext, G.R., 1963. The estimation of second-order tensors, with related tests and designs. Biometrika 50, 353-373. https://doi.org/10.2307/2333905.
Higgins, M.D., 2006. Quantitative Textural Measurements in Igneous and Metamorphic Petrology. Cambridge University Press, Cambridge. https://doi.org/10.1017/ CBO9780511535574.

Hirt, A.M., Almqvist, B.S.G., 2012. Unraveling magnetic fabrics. Int. J. Earth Sci. 101, 613-624. https://doi.org/10.1007/s00531-011-0664-0.

Islam, A., Chevalier, S., Sassi, M., 2018. Structural characterization and numerical simulations of flow properties of standard and reservoir carbonate rocks using microtomography. Comput. Geosci. 113, 14-22. https://doi.org/10.1016/j. cageo.2018.01.008.

Jelínek, V., 1981. Characterization of the magnetic fabric of rocks. Tectonophysics 79, T63-T67. https://doi.org/10.1016/0040-1951(81)90110-4.

Jelínek, V., 1978. Statistical processing of anisotropy of magnetic susceptibility measured on groups of specimens. Studia Geophys. Geod. 22, 50-62. https://doi. org/10.1007/BF01613632.

Jerram, D.A., Higgins, M.D., 2007. 3D analysis of rock textures: quantifying igneous microstructures. Elements 3, 239-245.

Ketcham, R.A., 2005a. Three-dimensional grain fabric measurements using highresolution X-ray computed tomography. J. Struct. Geol. 27, 1217-1228. https://doi. $\operatorname{org} / 10.1016 /$ j.jsg.2005.02.006.

Ketcham, R.A., 2005b. Computational methods for quantitative analysis of threedimensional features in geological specimens. Geosphere 1, 32-41. https://doi.org/ 10.1130/GES00001.1.

Ketcham, R.A., Carlson, W.D., 2001. Acquisition, optimization and interpretation of Xray computed tomographic imagery: applications to the geosciences. Comput. Geosci. 27, 381-400. https://doi.org/10.1016/S0098-3004(00)00116-3.

Launeau, P., Cruden, A.R., 1998. Magmatic fabric acquisition mechanisms in a syenite: results of a combined anisotropy of magnetic susceptibility and image analysis study. J. Geophys. Res. Solid Earth 103, 5067-5089. https://doi.org/10.1029/97JB02670.

Launeau, P., Robin, P.-Y.F., 2005. Determination of fabric and strain ellipsoids from measured sectional ellipses-implementation and applications. J. Struct. Geol. 27, 2223-2233. https://doi.org/10.1016/j.jsg.2005.08.003.

Launeau, P., Robin, P.-Y.F., 1996. Fabric analysis using the intercept method. Tectonophysics 267, 91-119. https://doi.org/10.1016/S0040-1951(96)00091-1.

Lisle, R.J., 1989. The statistical analysis of orthogonal orientation data. J. Geol. 97, 360-364.

Macente, A., Fusseis, F., Menegon, L., Xiao, X., John, T., 2017. The strain-dependent spatial evolution of garnet in a high- P ductile shear zone from the Western Gneiss Region (Norway): a synchrotron X-ray microtomography study. J. Metamorph. Geol. 35, 565-583. https://doi.org/10.1111/jmg.12245.

Mardia, K.V., Jupp, P.E., 2009. Directional Statistics. John Wiley \& Sons.

Moreno Chavez, G., Castillo Rivera, F., Sarocchi, D., Borselli, L., Rodríguez-Sedano, L.A., 2018. FabricS: a user-friendly, complete and robust software for particle shape-fabric analysis. Comput. Geosci. 115, 20-30. https://doi.org/10.1016/j. cageo.2018.02.005.

Nicolas, A., 1992. Kinematics in magmatic rocks with special reference to gabbros. J. Petrol. 33, 891-915. https://doi.org/10.1093/petrology/33.4.891.

Odgaard, A., 1997. Three-dimensional methods for quantification of cancellous bone architecture. Bone 20, 315-328. https://doi.org/10.1016/S8756-3282(97)00007-0.

Odgaard, A., Kabel, J., van Rietbergen, B., Dalstra, M., Huiskes, R., 1997. Fabric and elastic principal directions of cancellous bone are closely related. J. Biomech. 30, 487-495. https://doi.org/10.1016/S0021-9290(96)00177-7.

Petri, B., Skrzypek, E., Mohn, G., Mateeva, T., Robion, P., Schulmann, K., Manatschal, G., Müntener, O., 2018. Mechanical anisotropies and mechanisms of mafic magma ascent in the middle continental crust: the Sondalo magmatic system (N Italy). GSA Bull 130, 331-352. https://doi.org/10.1130/B31693.1.

Prior, D.J., Boyle, A.P., Brenker, F., Cheadle, M.C., Day, A., Lopez, G., Peruzzo, L., Potts, G.J., Reddy, S., ans Nick, E., Timms, R.S., Trimby, P., Wheeler, J., Zetterstrom, L., 1999. The application of electron backscatter diffraction and orientation contrast imaging in the SEM to textural problems in rocks. Am. Mineral. $84,1741-1759$.

Ramsay, J.G., 1967. Folding and Fracturing of Rock. McGraw-Hill, New York.

Robin, P.-Y.F., 2002. Determination of fabric and strain ellipsoids from measured sectional ellipses - theory. J. Struct. Geol. 24, 531-544. https://doi.org/10.1016/ S0191-8141(01)00081-5.

Scheidegger, A.E., 1965. On the statistics of the orientation of bedding planes, grain axes, and similar sedimentological data. U. S. Geol. Surv. Prof. Pap. 525, 164-167.

Schopa, A., Floess, D., de Saint Blanquat, M., Annen, C., Launeau, P., 2015. The relation between magnetite and silicate fabric in granitoids of the Adamello Batholith. Tectonophysics 642, 1-15. https://doi.org/10.1016/j.tecto.2014.11.022.

Shan, Y., 2008. An analytical approach for determining strain ellipsoids from measurements on planar surfaces. J. Struct. Geol. 30, 539-546. https://doi.org/ $10.1016 /$ j.jsg 2006.12.004.

Smit, Schneider, Odgaard, 1998. Star length distribution: a volume-based concept for the characterization of structural anisotropy. J. Microsc. 191, 249-257. https://doi.org/ 10.1046/j.1365-2818.1998.00394.x.

Som, S.M., Hagadorn, J.W., Thelen, W.A., Gillespie, A.R., Catling, D.C., Buick, R., 2013. Quantitative discrimination between geological materials with variable density contrast by high resolution X-ray computed tomography: an example using amygdule size-distribution in ancient lava flows. Comput. Geosci. 54, 231-238. https://doi.org/10.1016/j.cageo.2012.11.019.

Starnoni, M., Pokrajac, D., Neilson, J.E., 2017. Computation of fluid flow and pore-space properties estimation on micro-CT images of rock samples. Comput. Geosci. 106, 118-129. https://doi.org/10.1016/j.cageo.2017.06.009.

Tauxe, L., 2003. Paleomagnetic Principles and Practice, Modern Approaches in Geophysics. Kluwer Academic Publishers, Dordrecht. https://doi.org/10.1007/0306-48128-6. 
Titschack, J., Baum, D., Matsuyama, K., Boos, K., Farber, C., Kahl, W.-A., Ehrig, K., Meinel, D., Soriano, C., Stock, S.R., 2018. Ambient occlusion - a powerful algorithm to segment shell and skeletal intrapores in computed tomography data. Comput. Geosci. 115, 75-87. https://doi.org/10.1016/j.cageo.2018.03.007.

Ulrich, S., Mainprice, D., 2005. Does cation ordering in omphacite influence development of lattice-preferred orientation? J. Struct. Geol. 27, 419-431. https:// doi.org/10.1016/j.jsg.2004.11.003.

Vollmer, F.W., 1995. C program for automatic contouring of spherical orientation data using a modified Kamb method. Comput. Geosci. 21, 31-49. https://doi.org/ 10.1016/0098-3004(94)00058-3.

Vollmer, F.W., 1990. An application of eigenvalue methods to structural domain analysis. Geol. Soc. Am. Bull. 102, 786-791.

Vontobel, P., Lehmann, E.H., Hassanein, R., Frei, G., 2006. Neutron tomography: method and applications. Phys. B Condens. Matter 385-386, 475-480. https://doi.org/ 10.1016/j.physb.2006.05.252.
Watson, G.S., 1966. The statistics of orientation data. J. Geol. 74, 786-797.

Watson, G.S., Irving, E., 1957. Statistical methods in rock magnetism. Geophys. J. Int. 7, 289-300. https://doi.org/10.1111/j.1365-246X.1957.tb02882.x.

Werner, T., 1997. Experimental designs for determination of the anisotropy of remanence-test of the efficiency of least-square and bootstrap methods applied to metamorohic rocks from southern Poland. Phys. Chem. Earth 22, 131-136. https:// doi.org/10.1016/S0079-1946(97)00090-6.

Whitehouse, W.J., 1974. The quantitative morphology of anisotropic trabecular bone. J. Microsc. 101, 153-168, https://doi.org/10.1111/j.1365-2818.1974.tb03878.x.

Woodcock, N.H., 1977. Specification of fabric shapes using an eigenvalue method. Geol. Soc. Am. Bull. 88, 1231. https://doi.org/10.1130/0016-7606(1977)88<1231: SOFSUA $>2.0 . \mathrm{CO} ; 2$ 